\title{
Global Attenuation in Spiral Galaxies in Optical and Infrared Bands
}

\author{
Ehsan Kourkchi ${ }^{1}$ (D), R. Brent Tully ${ }^{1}$ (D) J. Don Neill $^{2}$, Mark Seibert ${ }^{3}$ (D) , Hélène M. Courtois ${ }^{4}$, and Alexandra Dupuy ${ }^{4}$ \\ ${ }^{1}$ Institute for Astronomy, University of Hawaii, 2680 Woodlawn Drive, Honolulu, HI 96822, USA; ehsan@ifa.hawaii.edu, tully@ifa.hawaii.edu \\ ${ }^{2}$ California Institute of Technology, 1200 East California Boulevard, MC 278-17, Pasadena, CA 91125, USA \\ ${ }^{3}$ The Observatories of the Carnegie Institute of Washington, 813 Santa Barbara Street, Pasadena, CA 91101, USA \\ ${ }^{4}$ University of Lyon, UCB Lyon 1, CNRS/IN2P3, IPN Lyon, France \\ Received 2019 April 23; revised 2019 August 30; accepted 2019 September 3; published 2019 October 14
}

\begin{abstract}
The emerging light from a galaxy is under the influence of its own interstellar medium, as well as its spatial orientation. Considering a sample of 2239 local spiral galaxies in optical (Sloan Digital Sky Survey $u, g, r, i$, and $z$ ) and infrared bands (WISE Wl,Wl), we study the dependency of the global intrinsic attenuation in spiral galaxies on their morphologies, sizes, and spatial inclinations. Reddening is minimal at the extremes of low mass and gas depletion and maximal in galaxies that are relatively massive and metal-rich and still retain substantial gas reserves. A principal component constructed from observables that monitor galaxy mass, relative H I content to old stars, and infrared surface brightness is strongly correlated with the amplitude of obscuration. We determine both a parametric model for dust obscuration and a nonparametric model based on the Gaussian process formalism. An average dust attenuation curve is derived for wavelengths between 0.36 and $4.5 \mu \mathrm{m}$.
\end{abstract}

Key words: galaxies: ISM - galaxies: photometry - galaxies: spiral

Supporting material: machine-readable table

\section{Introduction}

A large new data set provides insights into the properties of obscuration in spiral galaxies. The data have been accumulated toward the goal of determining galaxy distances through the correlation between the intrinsic luminosities and rotation rates of spiral galaxies (Tully \& Fisher 1977). It is important to understand the consequences of obscuration on observed luminosities for that purpose. In addition to the utilitarian use for the measurement of distances, there is a general interest in understanding the statistical properties of obscuration in spiral galaxies.

The light from stars is attenuated ${ }^{5}$ by intervening dust in the line of sight through a host galaxy. Dust accumulation is enhanced in metal-rich environments where the interstellar medium (ISM) has been retained. Attenuation of optical light is insignificant at the extremes of dwarf galaxies and galaxies overwhelmingly dominated by old stars. Dwarf galaxies can have considerable gas content, but most is in the atomic state, transparent to visible light. The gas in predominantly old systems has been efficiently transformed into stars or purged through winds or stripping. In between in terms of mass and evolution, there are normal spiral galaxies with orientations that can hide two-thirds of their stellar light at blueward passbands. It will be demonstrated that the degree of obscuration from a nonpathological galaxy is predictable, given a practical suite of observables.

The stellar light of galaxies is obscured through interactions with their interstellar gas and dust. The level of obscuration correlates with spatial orientation and physical properties of the host galaxy, including size or mass, morphology, formation history, and metallicity, or the chemical compositions of its interstellar environment (Draine 2011). The effect of dust

\footnotetext{
5 Following advice from the referee, we reserve the term "extinction" to refer to the local interplay of absorption of scattering of light with the ISM and use the term "attenuation" for the global effect dependent on source geometry and attendant path lengths.
}

obscuration is less pronounced at longer wavelengths, and on average it effectively shifts the galaxy color redward. The amplitude of dust obscuration in spirals increases with their inclination from face-on because interactions between the galaxy photons and its dust/gas particles occur along longer paths in the direction of the observer line of sight (Masters et al. 2010). There have been various attempts to study the influence of dust on the observed luminosity of spirals at multiple wavelengths (Giovanelli et al. 1995; Tully et al. 1998; Masters et al. 2003; Unterborn \& Ryden 2008). Cho \& Park (2009) describe dust attenuation in late-type galaxies in terms of a concentration index and $K s$-band absolute luminosity. Yip et al. (2010) study the dependency of dust attenuation in spirals on their orientations from measurements of Balmer line ratio, $\mathrm{H} \alpha / \mathrm{H} \beta$. Xiao et al. (2012) find an empirical relation between dust attenuation and such physical parameters of spirals as $\mathrm{H} \alpha$ luminosity, $\mathrm{H} \alpha$ surface brightness, metallicity, and axial ratio.

The information we bring to the problem begins with the kinematic information derived from global $21 \mathrm{~cm}$ neutral hydrogen profiles. H I line widths are a distance-independent proxy for intrinsic luminosities (Tully et al. 1998). The H I observations also provide fluxes. Pseudocolors formed from optical/infrared photometry and $\mathrm{HI}$ fluxes are distance independent and are correlated with morphology, stellar populations, interstellar gas content, and presumably the accumulation of dust.

In addition, we have multiband surface photometry, using the $u, g, r, i$, and $z$ bands from the archive of the Sloan Digital Sky Survey (SDSS) and the $W 1$ and $W 2$ (3.4 and $4.6 \mu \mathrm{m})$ bands from imaging with the Wide-field Infrared Survey Explorer (WISE). Colors formed by combinations of these bands are independent of distance and bear on stellar populations-ages and metallicities-as well as the effects of reddening. Photometry also provides two other distance-independent parameters that reflect the morphologies of galaxies: surface brightness and degree of central concentration. 
Finally, there is galaxy inclination, also a distanceindependent parameter. Cumulative obscuration increases sharply in galaxies toward the edge-on orientation. We have carefully measured the inclinations of our spirals by manually comparing them to a set of standard spirals with known inclinations. We believe that our measured inclinations are more accurate compared to those calculated from the axial ratio of photometry isodensity ellipses that are subject to morphological peculiarities, and in some cases they may represent the galaxy bulge instead of its spiral disk.

The problem posed to us is to disentangle the matrix of mutually correlated observables with colors from multiband optical, infrared, and H I fluxes on one axis of the matrix, and the structural parameters such as surface brightness and concentration, $\mathrm{H}$ I line width as a proxy for intrinsic luminosity, and inclinations on the other axis, using a sample of 2239 galaxies. We monitor the amplitude of obscuration as a function of stellar and gas properties as manifested by inclination dependencies. Our parameters are all distance independent, which enables an analysis with a sample large enough to characterize the dominant tendencies.

We present our data products in Section 2. In Section 3, we introduce a set of distance-independent observables, as well as the main principal component combination of observables that minimizes dispersion in optical-infrared colors, to address the problem of dust obscuration. In Section 4, we take parametric and nonparametric approaches to formulate the inclinationdependent dust attenuation. In Section 5 we calculate the average dust attenuation in spirals at different wavelengths. We summarize our results in Section 6.

\section{Data}

The sample for this study comprises 2239 spiral galaxies taken from a grand catalog of $\sim 20,000$ spiral galaxies ${ }^{6}$ assembled for the measurement of distances using the correlation between galaxy H I line widths and luminosities known as the TullyFisher relationship (TFR; Tully \& Fisher 1977). Galaxies of this grand catalog were selected based on the following criteria: (1) All lie within $15,000 \mathrm{~km} \mathrm{~s}^{-1}$, most within the decl. range accessible to the Arecibo Telescope of $0<\delta<+38$. (2) Morphological types are Sa or later. (3) Their preliminarily estimated inclinations are greater than $45^{\circ}$ from face-on based on axial ratios taken from HyperLEDA ${ }^{7}$ (Paturel et al. 2003). (4) All are restricted to have high-quality $\mathrm{H}$ I measurements as explained in Section 2.1.

The current study of dust attenuation requires spirals to have both optical and infrared photometry coverage that is not currently available for the full sample of $\sim 20,000$ galaxies. We have completed the optical photometry of all galaxies with SDSS coverage, but the infrared photometry of the full sample was not completed at the time of this study. The requirement of complete photometry in all bands (optical and infrared) left us with a subsample of 2239 galaxies for use in this study.

The neutral hydrogen line widths and fluxes, sources, and criteria used for the compilation of our sample are discussed in Section 2.1. Our procedures to obtain and analyze optical and infrared images are explained in Sections 2.2-2.4. Regarding inclinations, axial ratios are not sufficiently accurate indicators of spiral inclinations, so we only used such estimates to give a

\footnotetext{
6 This catalog will be presented in a following paper.

7 http://leda.univ-lyon1.fr/
}

preliminary sample selection cut at $45^{\circ}$. More face-on spirals are excluded because uncertainties in line width deprojection become prohibitive. In Section 2.5 we discuss how we proceeded to acquire inclinations of reasonable quality and that supersede inclinations derived from axial ratios. In Section 2.6, we present our data catalog.

\subsection{Neutral Hydrogen}

$\mathrm{H}$ I line width and flux measurements of sufficient quality are provided by the All Digital H I catalog (ADHI) that has been assembled in the context of the Cosmicflows program (Tully et al. 2016) and is available at the Extragalactic Distance Database (EDD) website $^{8}$ and/or the Arecibo Legacy Fast ALFA Survey (ALFALFA; Haynes et al. 2011, 2018). The ADHI catalog provides $W_{m x}$, H I line widths that represent positive and negative extremes of galaxy rotation velocities, a parameter derived from the directly observed $W_{m 50}$, the line width at $50 \%$ of the mean $\mathrm{H}$ I flux within the area that contains 90\% of the total H I flux (Courtois et al. 2011; Courtois \& Tully 2012; Tully \& Courtois 2012). We adjust ALFALFA line widths, $W_{\text {alf }}$, for compatibility with the ADHI values using $W_{m x}=W_{\text {alf }}-6 \mathrm{~km} \mathrm{~s}^{-1}$, which we derived for galaxies covered by both catalogs. We use a weighted average of $\mathrm{HI}$ measurements if a galaxy is included in both catalogs. Our interest is in spiral, not dwarf, galaxies, so we set a threshold of $W_{m x}>64 \mathrm{~km} \mathrm{~s}^{-1}$. To avoid marginal data, we require $\mathrm{S} / \mathrm{N}>10$ for line widths taken from the ALFALFA catalog and we only accept ADHI $W_{m x}$ values with uncertainties less than or equal to $20 \mathrm{~km} \mathrm{~s}^{-1}$. These two criteria are chosen to be fairly consistent for a set of common galaxies in both catalogs. Coupled with the availability of high-quality optical/infrared photometry and inclination measurements, we draw 2004 galaxies from ADHI and 1248 galaxies from ALFALFA, many in common. A further $61 \mathrm{HI}$ line width measurements were extracted from Springob et al. (2005), where their line width values $W_{M 50}$ are adjusted to our $W_{m 50}$ using the linear relationship $W_{m 50}-W_{M 50}=1.015 W_{m 50}-11$ (Courtois et al. 2009). To summarize, out of the final 2239 spirals in our sample, 61 have H I measurements from Springob et al. (2005), and the rest are covered by ADHI and/or ALFALFA catalogs.

$\mathrm{H}$ I fluxes are derived from the average of all available fluxes drawing on these resources. Analogous to the magnitude scale used to characterize optical and near-infrared fluxes, the $21 \mathrm{~cm}$ line flux translates to the magnitude $m_{21}$ with the relation

$$
m_{21}=-2.5 \log F_{\mathrm{HI}}+17.40
$$

where $F_{\mathrm{H} \text { I }}$ is the area of the $21 \mathrm{~cm}$ line profile in units of Jy $\mathrm{km} \mathrm{s}^{-1}$.

\subsection{Optical Images}

To obtain the cutout image of each galaxy at $u, g, r, i$, and $z$ bands, we download all corresponding calibrated single exposures from the SDSS DR12 database (York et al. 2000). All exposures have already been calibrated and sky subtracted. Therefore, we do not perform any sky subtraction prior to combining frames. We use MONTAGE, a toolkit for assembling astronomical images (Jacob et al. 2010), to drizzle all frames and construct galaxy images. The angular scale of the output images is $0.4^{\prime \prime} \mathrm{pixel}^{-1}$. Our data acquisition pipeline is

\footnotetext{
8 http://edd.ifa.hawaii.edu; catalog "All Digital H I."
} 
accessible online. ${ }^{9}$ SDSS provides images that are calibrated in such a way that all measured magnitudes are supposed to be in the AB system in all ugriz bands. Although this is correct for gri bands, there are small departures for SDSS $u$ - and $z$-band zero-points from the $\mathrm{AB}$ system that are corrected using $u_{A B}=u_{\mathrm{SDSS}}-0.04 \mathrm{mag}$ and $z_{A B}=z_{\mathrm{SDSS}}+0.02 \mathrm{mag} .{ }^{10}$

\subsection{Infrared Images}

We use the W1 $(3.4 \mu \mathrm{m})$ and W2 $(4.6 \mu \mathrm{m})$ images of the WISE survey (Wright et al. 2010). All WISE images are available to the public through the NASA/IPAC Infrared Science Archive (IRSA). Following the same procedure explained by Neill et al. (2014), image products were constructed by drizzling singleexposure images using version 3.8.4 of the Image Co-addition with Optional Resolution Enhancement (ICORE) software (Masci \& Fowler 2009; Masci 2013). All of our final co-added images have a spatial scale of $1^{\prime \prime}$ pixel $^{-1}$. To reduce uncertainties due to background subtraction, images within $25^{\circ}$ of the Moon and within $2000 \mathrm{~s}$ of an annealing event were avoided. To improve the image depth, we also use images from the Neo-WISE project, which is the extended WISE mission to find asteroids. We limit the total number of co-added frames to 200 to keep the processing time manageable. The depth of the final cutouts depends on the position of a target relative to the ecliptic plane, with coverage density larger at the ecliptic poles and smaller close to the ecliptic plane (Wright et al. 2010). We used Vega-AB offsets of 2.699 mag for $W 1$ and 3.339 mag for $W 2$ to transform from the Vega to the $\mathrm{AB}$ system following Table 3 of Section IV.4.h of the Explanatory Supplement to the WISE All-Sky Data Release Products. ${ }^{11}$

\subsection{Photometry}

Photometry was performed using the modified version of the photometry pipeline originally developed to generate the WISE Nearby Galaxy Atlas (WNGA; M. Seibert et al. 2019, in preparation). The process starts with taking all geometrical information from the HyperLEDA catalog to define initial photometry apertures that are later modified either by visual inspection or with the aid of SExtractor (Bertin \& Arnouts 1996) or DS9. ${ }^{12}$ We estimate the sky background level within an annulus far from an aperture enclosing the galaxy. All foreground Galactic stars and overlapping objects are masked. The light profile of each galaxy is calculated within concentric elliptical annuli with increments of $3^{\prime \prime}$ for $u$, $g, r, i$, and $z$ bands and $6^{\prime \prime}$ for $W 1$ and $W 2$ bands. Resulting light profiles and growth curves are visually inspected, and further masking and annulus adjustments are applied if necessary. The entire process is repeated until the growth curves converge.

The sky level of our SDSS cutouts is minimal because images have been sky subtracted. On the infrared side, the large resolution elements of $W 1$ and $W 2$ images $\left(\sim 6^{\prime \prime}\right)$ make it more challenging to estimate accurate sky values. Faint unresolved and therefore unmasked galaxies and foreground stars in the sky annulus influence the quality of photometry results. These issues are addressed by visual inspections, as well as control of masking limits. For most galaxies in our sample, we adopt the same recipe explained in Neill et al. (2014) for the photometry

\footnotetext{
9 https://github.com/ekourkchi/SDSS_get

10 https://www.sdss.org/dr12/algorithms/fluxcal/

11 http://wise2.ipac.caltech.edu/docs/release/allsky/expsup/sec4_4h.html

12 http://ds9.si.edu/site/Home.html
}

of W1 and W2 images and the measurement of the sky level. In addition, for galaxies with a nonconvergent curve of growth, iterative microadjustments to the background level are evaluated to avoid surface brightness flares or drops and that allow the curve of growth to converge. The adjustment levels are a few percent of the initial estimations of the sky background that were judged within the background annulus.

For each galaxy, the WNGA pipeline derives radial light profiles and provides two versions of "total magnitude": (1) The asymptotic magnitude is calculated within the aperture radius at the point where the cumulative luminosity curve of growth flattens. Asymptotic magnitudes are robust since they do not depend on the aperture size chosen by the user. (2) An isophotal magnitude is defined within $25.5 \mathrm{mag} \operatorname{arcsec}^{-2}$ and then augmented with an extrapolation calculated from the extension of an exponential fit of the galaxy disk to infinity (Tully et al. 1996; Neill et al. 2014). The average discrepancy between these two types of magnitude is at most 0.02 mag in all bands. The standard deviation of the scatter is $\sim 0.02 \mathrm{mag}$ for the brightest galaxies, increasing with fainter objects; however, on average it remains below $0.05 \mathrm{mag}$ for all wavebands except the lower-quality $u$ band $(\sigma \sim 0.1 \mathrm{mag}$ ). Although the results of this study are insensitive to the chosen magnitude, we prefer asymptotic magnitudes to avoid any assumptions about galaxy type.

For each waveband, $\lambda$, the measured total magnitude of each galaxy, $m_{\lambda}^{\text {total }}$, is corrected for obscuration in the Milky Way, redshift with respect to each passband ( $k$-correction), and aperture effects, but not host obscuration, using the following relation:

$$
\bar{m}^{\lambda}=m_{\text {total }}^{\lambda}-A_{b}^{\lambda}-A_{k}^{\lambda}-A_{a}^{\lambda},
$$

where $A_{b}^{\lambda}$ is the Milky Way extinction calculated from $R_{\lambda} E(B-V), A_{k}^{\lambda}$ is the $k$-correction due to Doppler shift, and $A_{a}^{\lambda}$ is the total flux aperture correction. We use Schlegel et al. (1998) $100 \mu \mathrm{m}$ cirrus maps to extract Milky Way $E(B-V)$ values. For $u, g, r, i$, and $z$ bands, $R_{u, g, r, i, z}$ values are given by Schlafly \& Finkbeiner (2011). $R_{W 1}$ and $R_{W 2}$ coefficients are 0.186 and 0.123 , respectively (Fitzpatrick 1999). The $k$-corrections are very small over the redshift range of interest. At optical bands, $k$-corrections are derived following the procedure presented by Chilingarian et al. (2010). For Wl and $W 2, k$-corrections are roughly the same and independent of galaxy type and follow a linear form $A_{k}^{W 1,2}=-2.27 z$ (Oke \& Sandage 1968; Huang et al. 2007). Aperture corrections are applied on the WISE magnitudes where point sources were used for photometric calibration within fixed apertures with the radius of $\sim 8.25^{\prime \prime}$, which is much smaller than our sample galaxies. As a result, the photometry from point-source calibrations must be corrected to account for the scattered diffuse light from extended sources. For each galaxy, we apply fixed corrections on $W 1$ and $W 2$ magnitudes, i.e., $A_{a}^{W 1}=-0.034$ and $A_{a}^{W 2}=-0.041 \mathrm{mag}$, taken from Table 5 of Section IV.4.c of the WISE Explanatory Supplement. ${ }^{13}$

\subsection{Galaxy Inclinations}

The inclinations of spiral galaxies can be coarsely derived from the ellipticity of apertures used for photometry, assuming

\footnotetext{
${ }^{13}$ http://wise2.ipac.caltech.edu/docs/release/allsky/expsup/sec4_4c.html
} 
that the image of a spiral galaxy is the projection of a disk with the shape of an oblate spheroid (Tully \& Pierce 2000). For $\sim 1 / 3$ of spiral galaxies the approximation of axial ratios provides inclination estimates good to better than $5^{\circ}$, with degradation to $\sim 5^{\circ}$ for another $1 / 3$. However, in $\sim 1 / 3$ of cases, ellipticity-derived inclinations are problematic for a variety of reasons. Prominent bulges can dominate the axial ratio measurement, with the Sombrero galaxy (NGC 4594) providing an extreme example. Some galaxies may not be axially symmetric owing to tidal effects. High surface brightness bars within much lower surface brightness disks can lead to large errors. Simply the orientation of strong spiral features with respect to the tilt axis (spirals opening onto the minor vs. the major axis) can be confusing. The statistical derivation of inclinations for large samples has been unsatisfactory. With great effort, galaxy images can be broken into multiple components, isolating the disk (Salo et al. 2015). While such an analysis can give inclinations accurate to $2^{\circ}-3^{\circ}$ in clean cases, in many cases, especially in samples of tens of thousands of galaxies with images that degrade with distance, uncertainties can be unsatisfactorily large. These problems compel a new approach.

We have investigated the capability of the human eye to evaluate galaxy inclinations. We begin with the advantage that a substantial fraction of spiral inclinations are well defined by axial ratios. These good cases give us a grid of standards of wide morphological types over the inclination range that particularly interests us of $45^{\circ}-90^{\circ}$. The challenge is to fit random target galaxies into the grid, thus providing estimates of their inclinations.

To achieve our goal, we designed an online graphical tool, Galaxy Inclination Zoo, ${ }^{14}$ to measure the inclination of spiral galaxies in our sample. In this graphical interface, we use the colorful images provided by SDSS, as well as the $g-, r$-, and $i$-band images generated for our photometry program. The latter are presented in black and white after rescaling by the $a$ sinh function to differentiate more clearly the internal structures of galaxies. The inclinations of standard galaxies were initially measured based on their $I$-band axial ratios (Tully \& Courtois 2012). Each galaxy is compared with the standard galaxies in two steps. First, the user locates the galaxy among nine standard galaxies sorted by their inclinations ranging between $45^{\circ}$ and $90^{\circ}$ in increments of $5^{\circ}$. In step two, the same galaxy is compared with nine other standard galaxies whose inclinations are $1^{\circ}$ apart and cover the $5^{\circ}$ interval found in the first step. At the end, the inclination is calculated by averaging the inclinations of the standard galaxies on the left/right side of the target galaxy. In the first step, if a galaxy is classified to be more face-on than $45^{\circ}$, it is flagged and step two is skipped.

Initially, the tool was tested by two of the authors (E.K. looked at essentially all of the candidates, and R.B.T. gave attention to about half). The tool was further tested with the participation of 10 undergraduate students and friends, with multiple cross-checks allowing the assessment of consistency and potentially revealing systematic differences. The program was then opened to the public and hence benefited from the participation of citizen scientists and tens of amateur astronomers. Ultimately, for each spiral we use all the measured inclinations by all users who worked on that galaxy.

We take the following precautions to minimize userdependent and user-independent biases: (1) We round the

\footnotetext{
${ }^{14}$ http://edd.ifa.hawaii.edu/inclination/index.php
}

resulting inclinations to the next highest or smallest integer values chosen randomly. (2) At each step, standard galaxies are randomly drawn with an option for users to change them randomly to verify their work or to compare galaxies with similar structures. (3) To increase the accuracy of the results, we catalog the median of at least three different measurements performed by different users. (4) Users may reject galaxies for various reasons and leave comments with the aim of avoiding dubious cases.

The uncertainties on the measured inclinations are estimated based on the statistical scatter in the reported values by different users. We believe that we have achieved a statistical accuracy of $\pm 4^{\circ}$ rms. A more detailed discussion of these measurements and their uncertainties will be given in an upcoming data paper (E. Kourkchi et al. 2019, in preparation).

An obvious step forward would be to replace the human eye with a machine learning algorithm. Such an endeavor would require an instruction set of order $10^{4}$ representative galaxies with reasonably established inclinations. Our entire sample is of comparable size to the required training set. Machine learning will be called for with larger samples in the future.

\subsection{Data Catalog}

The results of our measurements and the collection of other parameters for 2239 spiral galaxies we used in this study are gathered in Table 1. Columns of this table include the following: Column (1) gives the Principal Galaxies Catalog (PGC) number and Column (2) the common name. Column (3) is $\mathrm{H} \mathrm{I}$ line width in $\mathrm{km} \mathrm{s}^{-1}$ and Column (4) is the logarithm of the inclination-corrected $\mathrm{HI}$ line width, calculated from $W_{m x}^{i}=W_{m x} / \sin (i)$, where $i$ is the inclination angle presented in Column (13). Column (5) is H I $21 \mathrm{~cm}$ magnitude calculated from the $\mathrm{HI}$ flux, $F_{\mathrm{H} \text { I }}$, using $m_{21}=-2.5 \log F_{\mathrm{HI}}+17.4$. Columns (6)-(10) are the $\operatorname{SDSS} u, g, r, i$, and $z$ total magnitudes in the AB system. Columns (11)-(12) are WISE WI and $W 2$ total magnitudes in the $\mathrm{AB}$ system. Except for the $u$ band, the uncertainties on all the measured magnitudes are not worse than $0.05 \mathrm{mag}$, the value we adopt conservatively for error propagation in this study. For the $u$-band magnitudes, uncertainties are $\sim 0.1$ mag. Column (13) stores our measured inclinations, with empty cells for galaxies with inclinations less than $45^{\circ}$ (see Section 2.5). Column (14) gives the half-light radii measured at $W 2$ bands. Column (15) holds the concentration index that is defined as $C_{82}=5 \log \left(r_{80} / r_{20}\right)$, where $r_{80}$ and $r_{20}$ are the semimajor axes of the isophotes enclosing $80 \%$ and $20 \%$, respectively, of the total galaxy light at the $W 2$ band (Kent 1985). Column (16) contains the axial ratio, $b / a$, of the elliptical apertures that we used for the photometry of W1(2)-band images, where $a$ and $b$ are the aperture semimajor and semiminor axes. Columns (17)-(23) tabulate optical/infrared magnitudes corrected for Milky Way obscuration, redshift $k$-correction, and aperture effects, but not host inclination, following Equation (2), based on the corresponding raw magnitudes listed in Columns (6)-(12).

\section{Intrinsic Obscuration in Spiral Galaxies}

The observed light from the stellar content of a galaxy depends on interactions with its own ISM. The level of flux attenuation depends on the light wavelength and the physical nature of the ISM, as well as the orientation of the galaxy relative to the observer line of sight. The composition of the 
(This table is available in its entirety in machine-readable form.)

Table 1

Data Catalog

\begin{tabular}{|c|c|c|c|c|c|c|c|c|c|c|c|c|c|c|c|c|c|c|c|c|c|c|}
\hline PGC & $\begin{array}{l}\text { Name } \\
\text { (2) }\end{array}$ & $\begin{array}{c}W_{m x} \\
\left(\mathrm{~km} \mathrm{~s}^{-1}\right) \\
(3)\end{array}$ & $\begin{array}{c}\log \left(W_{m x}^{i}\right) \\
(4)\end{array}$ & $\begin{array}{c}m_{21} \\
(\mathrm{mag}) \\
(5)\end{array}$ & $\begin{array}{c}\begin{array}{c}u \\
\text { (mag) } \\
(6)\end{array} \\
\text { (a) }\end{array}$ & $\begin{array}{c}g \\
(\mathrm{mag}) \\
(7)\end{array}$ & $\begin{array}{c}r \\
(\mathrm{mag}) \\
(8)\end{array}$ & $\begin{array}{c}i \\
(\mathrm{mag}) \\
(9)\end{array}$ & $\begin{array}{c}z \\
(\mathrm{mag}) \\
(10)\end{array}$ & $\begin{array}{c}W 1 \\
\text { (mag) } \\
(11)\end{array}$ & $\begin{array}{c}W 2 \\
(\mathrm{mag}) \\
(12)\end{array}$ & $\begin{array}{c}\text { Inc. } \\
\text { (deg) } \\
(13)\end{array}$ & $\begin{array}{c}R_{e} \\
(\operatorname{arcmin}) \\
(14)\end{array}$ & (15) & (16) & $\begin{array}{c}\bar{u} \\
(\mathrm{mag}) \\
(17)\end{array}$ & $\begin{array}{c}\bar{g} \\
\text { (mag) } \\
(18)\end{array}$ & $\begin{array}{c}\bar{r} \\
(\mathrm{mag}) \\
(19)\end{array}$ & $\begin{array}{c}\bar{i} \\
(\mathrm{mag}) \\
(20)\end{array}$ & $\begin{array}{c}\underset{z}{\bar{m}} \\
(21)\end{array}$ & $\begin{array}{c}\overline{W 1} \\
\text { (mag) } \\
(22)\end{array}$ & $\begin{array}{c}\overline{W 2} \\
\text { (mag) } \\
(23)\end{array}$ \\
\hline 4 & PGC 000004 & $154 \pm 3$ & $2.189 \pm 0.011$ & $16.69 \pm 0.08$ & 17.57 & 16.43 & 15.91 & 15.57 & 15.42 & 16.06 & 16.42 & $86 \pm 2$ & 0.33 & 3.34 & 0.33 & 17.11 & 16.11 & 15.69 & 15.41 & 15.30 & 16.08 & 16.44 \\
\hline 16 & PGC 000016 & $296 \pm 14$ & $2.515 \pm 0.025$ & $17.21 \pm 0.18$ & 16.08 & 14.83 & 14.21 & 13.86 & 13.61 & 13.98 & 14.67 & $65 \pm 4$ & 0.20 & 2.66 & 0.64 & 15.86 & 14.69 & 14.12 & 13.79 & 13.56 & 14.02 & 14.71 \\
\hline 55 & UGC 12898 & $179 \pm 10$ & $2.264 \pm 0.025$ & $15.76 \pm 0.08$ & 16.82 & 16.08 & 15.71 & 15.50 & 15.40 & 16.18 & 16.60 & $77 \pm 3$ & 0.26 & 2.76 & 0.46 & 16.53 & 15.90 & 15.58 & 15.42 & 15.33 & 16.21 & 16.63 \\
\hline 68 & ESO 538-017 & $206 \pm 18$ & $2.395 \pm 0.043$ & $16.88 \pm 0.18$ & 16.31 & 15.24 & 14.86 & 14.69 & 14.49 & 14.99 & 15.49 & $56 \pm 4$ & 0.19 & 3.15 & 0.66 & 16.12 & 15.15 & 14.79 & 14.67 & 14.46 & 15.04 & 15.54 \\
\hline 70 & UGC 12900 & $432 \pm 2$ & $2.636 \pm 0.003$ & $15.05 \pm 0.08$ & 16.70 & 15.41 & 14.66 & 14.25 & 13.87 & 13.55 & 14.08 & $90 \pm 1$ & 0.43 & 3.10 & 0.14 & 16.27 & 15.10 & 14.46 & 14.10 & 13.76 & 13.59 & 14.12 \\
\hline 76 & UGC 12901 & $390 \pm 4$ & $2.624 \pm 0.013$ & $15.78 \pm 0.08$ & 15.83 & 14.39 & 13.65 & 13.26 & 13.00 & 13.21 & 13.77 & $68 \pm 4$ & 0.32 & 3.57 & 0.47 & 15.52 & 14.18 & 13.51 & 13.16 & 12.93 & 13.25 & 13.82 \\
\hline 102 & IC 5376 & $427 \pm 6$ & $2.635 \pm 0.007$ & $15.41 \pm 0.08$ & 15.81 & 14.28 & 13.45 & 13.02 & 12.73 & 12.85 & 13.45 & $82 \pm 3$ & 0.34 & 4.26 & 0.27 & 15.42 & 14.00 & 13.27 & 12.88 & 12.63 & 12.87 & 13.48 \\
\hline 117 & IC 1526 & $193 \pm 19$ & $2.478 \pm 0.142$ & $17.43 \pm 0.08$ & 15.86 & 14.60 & 13.95 & 13.58 & 13.37 & 13.45 & 13.97 & & 0.18 & 2.99 & 0.88 & 15.44 & 14.31 & 13.76 & 13.44 & 13.27 & 13.51 & 14.03 \\
\hline 118 & PGC 000118 & $113 \pm 6$ & $2.248 \pm 0.138$ & $16.49 \pm 0.08$ & 16.36 & 15.46 & 15.05 & 14.82 & 14.66 & 15.10 & 15.74 & & 0.23 & 3.32 & 0.94 & 16.07 & 15.28 & 14.92 & 14.75 & 14.60 & 15.14 & 15.78 \\
\hline 124 & UGC 12913 & $251 \pm 5$ & $2.402 \pm 0.010$ & $15.78 \pm 0.08$ & 17.23 & 15.85 & 15.26 & 14.96 & 14.76 & 15.18 & 15.75 & $85 \pm 2$ & 0.34 & 3.41 & 0.29 & 17.04 & 15.74 & 15.18 & 14.91 & 14.72 & 15.22 & 15.79 \\
\hline 146 & UGC 12916 & $162 \pm 2$ & $2.325 \pm 0.027$ & $16.72 \pm 0.08$ & 16.80 & 15.72 & 15.22 & 14.92 & 14.82 & 15.43 & 16.02 & $50 \pm 4$ & 0.27 & 3.14 & 0.74 & 16.59 & 15.60 & 15.13 & 14.87 & 14.78 & 15.47 & 16.06 \\
\hline 155 & PGC 000155 & $212 \pm 20$ & $2.351 \pm 0.042$ & $17.60 \pm 0.08$ & 16.92 & 15.84 & 15.28 & 14.97 & 14.74 & 15.01 & 15.45 & $71 \pm 3$ & 0.17 & 2.48 & 0.60 & 16.44 & 15.51 & 15.05 & 14.82 & 14.62 & 15.05 & 15.50 \\
\hline 165 & UGC 12920 & $274 \pm 1$ & $2.445 \pm 0.005$ & $16.22 \pm 0.08$ & 16.67 & 15.59 & 15.06 & 14.75 & 14.58 & 14.78 & 15.14 & $81 \pm 3$ & 0.24 & 3.06 & 0.47 & 16.37 & 15.39 & 14.92 & 14.66 & 14.51 & 14.83 & 15.19 \\
\hline 176 & PGC 000176 & $349 \pm 13$ & $2.630 \pm 0.027$ & $16.08 \pm 0.08$ & 15.29 & 14.13 & 13.54 & 13.21 & 13.05 & 13.19 & 13.74 & $55 \pm 4$ & 0.24 & 3.19 & 0.60 & 15.03 & 13.96 & 13.43 & 13.13 & 12.99 & 13.23 & 13.78 \\
\hline 179 & PGC 000179 & $289 \pm 20$ & $2.462 \pm 0.030$ & $17.09 \pm 0.08$ & 16.74 & 15.34 & 14.50 & 14.05 & 13.78 & 13.84 & 14.33 & $86 \pm 2$ & 0.25 & 3.44 & 0.27 & 16.38 & 15.08 & 14.33 & 13.92 & 13.69 & 13.87 & 14.36 \\
\hline 185 & IC 5379 & $133 \pm 20$ & $2.167 \pm 0.067$ & $17.78 \pm 0.08$ & 17.07 & 15.96 & 15.46 & 15.17 & 14.99 & 15.60 & 16.21 & $65 \pm 4$ & 0.19 & 2.96 & 0.62 & 16.83 & 15.81 & 15.36 & 15.11 & 14.94 & 15.64 & 16.25 \\
\hline 186 & UGC 00003 & $472 \pm 4$ & $2.707 \pm 0.013$ & $15.37 \pm 0.08$ & 15.98 & 14.30 & 13.52 & 13.10 & 12.87 & 13.10 & 13.70 & $68 \pm 4$ & 0.28 & 3.62 & 0.54 & 15.71 & 14.13 & 13.42 & 13.02 & 12.81 & 13.15 & 13.75 \\
\hline 201 & UGC 00004 & $302 \pm 19$ & $2.572 \pm 0.035$ & $17.04 \pm 0.08$ & 16.07 & 14.84 & 14.25 & 13.89 & 13.66 & 13.99 & 14.58 & $54 \pm 4$ & 0.20 & 3.20 & 0.69 & 15.84 & 14.70 & 14.16 & 13.83 & 13.61 & 14.05 & 14.64 \\
\hline 205 & UGC 00005 & $446 \pm 20$ & $2.697 \pm 0.024$ & $15.63 \pm 0.08$ & 15.10 & 13.74 & 13.08 & 12.72 & 12.49 & 12.61 & 13.13 & $64 \pm 4$ & 0.30 & 2.92 & 0.56 & 14.83 & 13.56 & 12.96 & 12.64 & 12.43 & 12.66 & 13.18 \\
\hline 212 & IC 5381 & $677 \pm 11$ & $2.838 \pm 0.008$ & $15.99 \pm 0.08$ & 16.16 & 14.55 & 13.70 & 13.23 & 12.92 & 13.04 & 13.68 & $80 \pm 3$ & 0.26 & 3.29 & 0.42 & 15.82 & 14.32 & 13.56 & 13.12 & 12.85 & 13.12 & 13.76 \\
\hline
\end{tabular}


ISM depends on the type, mass, age, metallicity, and evolutionary history of a galaxy (Inoue 2011). Galaxy light is altered through the absorption of ultraviolet/visible photons by the ISM dust particles depending on dust grain size and emission of the received energy at longer wavelengths. Increasing the galaxy inclination, photons have a longer path length through the ISM, and therefore the obscuration effect is more pronounced. Accordingly, it is crucial to correct for obscuration when we use the TFR to measure distances of galaxies that are preferably chosen to have larger inclinations. There is the trade-off that kinematic errors diminish toward edge-on orientation while photometric errors increase. As a practical matter, it is more important to control the kinematic errors.

Understanding all physical processes involved in the intrinsic extinction of galaxies requires a much more detailed analysis that is beyond the scope of this study. Here, our main goal is to empirically estimate the inclination-dependent part of dust obscuration in spirals by correlating the obscuration with various distance-independent observable parameters representing different properties of galaxies. We list the observable parameters in the following: (1) The $\mathrm{H} \mathrm{I}$ line width corrected for inclination, $\log \left(W_{m x}^{i}\right)$, is related to the absolute magnitude of a galaxy via the TFR and therefore is a good proxy for the galaxy mass/size. (2) The optical-infrared colors $\left(\bar{m}_{\lambda}-\bar{W} j\right)$ characterize the attenuation of optical fluxes through the known property that the dust obscuration diminishes as wavelength increases until it is ultimately very tiny or negligible at infrared bands. (3) Another useful parameter is the pseudocolor difference between $21 \mathrm{~cm}$ and infrared magnitudes that probes $\mathrm{H}$ I to stellar flux, defined as

$$
C_{21 W j}=m_{21}-\bar{W} j,
$$

where $m_{21}$ is the H I $21 \mathrm{~cm}$ magnitude and $\bar{W} j$ is either the $\bar{W} 1$ or the $\bar{W} 2$ magnitude. Since $C_{21 W j}$ is constructed based on fluxes at infrared and longer wavelengths, it should be free of obscuration effects and an accurate proxy for the type of galaxy. In the absence of many active star-forming regions, galaxies with more negative (or bluer) $C_{21 \mathrm{Wj}}$ colors have larger $\mathrm{H}$ I to stellar mass ratio and vice versa. There is the caveat that in large star-forming spirals the infrared emission from hot dust near the star-forming regions and stochastic heating of small grains in diffuse regions augment the observed infrared fluxes (Law et al. 2018). In spirals with many star-forming regions, $W_{j}$ might be a poor proxy for stellar mass content such that $C_{21 W j}$ underestimates the ratio of $\mathrm{H}$ I to stellar mass. (4) The average surface brightness of galaxies is another distance-independent parameter that characterizes the galaxy type and its morphology. In this study, we use the effective surface brightness parameter calculated within the half-light radius in $\bar{W} 1 / \bar{W} 2$ infrared bands, $\left\langle\mu_{j}\right\rangle_{e}$. We correct the effective surface brightness to compensate for the effect of galaxy inclination using

$$
\left\langle\mu_{j}\right\rangle_{e}^{(i)}=\left\langle\mu_{j}\right\rangle_{e}+0.5 \log (a / b),
$$

where $a / b$ is the axial ratio of the elliptical aperture used for photometry. (5) The galaxy light concentration index, $C_{82}$, is another distance-independent photometry product that encodes bulge versus disk morphological information that might be useful in our analysis.

\subsection{Fiducial Correlations: Face-on Spirals}

Prior to examining the inclination-dependent part of attenuation, we first need to establish inclination-free fiducial correlations between optical-infrared colors and other distanceindependent observables. Then, we can attribute any deviations from the fiducial relations to the inclination of the galaxies. To establish the fiducial relations, in this section we restrict our sample to the more face-on spirals where the effect of inclination on attenuation will be at a minimum. To this end, we give attention to the 225 spirals in our sample that were flagged to be more face-on than $45^{\circ}$ by our more accurate inclination determination (Section 2.5). We arbitrarily set the inclination to be $40^{\circ} \pm 5^{\circ}$ for all of these galaxies to be able to perform our analysis in this section and to study the useful strong correlations between different observables. Although the inclinations of these nearly face-on galaxies are not exactly zero, their obscuration relative to face-on is minimal. Once we formulate the empirical fiducial model for face-on spirals, we no longer use these 225 nearly face-on galaxies in the numerical optimization of our model.

Figure 1 illustrates the correlation between $\bar{r}-\bar{W} 2$ color and different observable features introduced above for the 225 nearly face-on galaxies. Each point in this diagram represents a galaxy, color-coded based on the parameter $C_{21 W 2}$, with blue to red proceeding from gas prevalent to gas depleted. The typical error bar of the scattered points is given in the upper left corner of each panel. The rms is the root mean squared of deviations from the fitted dashed lines along the horizontal axis. Panels of this diagram are sorted based on the correlations between the plotted parameters, with panel (e) showing the weakest correlation between color and concentration parameters.

Panel (d) displays the correlation between color and $\log \left(W_{m x}^{i}\right)$. In this plot, the relative position of points and hence our final conclusions are insensitive to the arbitrarily chosen $40^{\circ} \pm 5^{\circ}$ for the inclination when estimating $\log \left(W_{m x}^{i}\right)$. True values of inclinations statistically shuffle points vertically on the $\log \left(W_{m x}^{i}\right)$ axis and only weakly affect the general correlation. The correlation between $\log \left(W_{m x}^{i}\right)$ and color is related to a color-magnitude correlation given that more massive, brighter galaxies have $\operatorname{larger} \log \left(W_{m x}^{i}\right)$ values. Larger galaxies tend to have older mean stellar populations, and as a result their optical-infrared colors are redder.

The observed correlation in panel (d) is not very strong (correlation coefficient equals 0.44). We are motivated to explore the possible role of other parameters to explain the scatter of galaxies around the fitted straight line. It is seen that almost all red points are distributed on the right side of the fitted line and most blue points are on its left side in panel (d). The red points represent galaxies with more positive $C_{21 W 2}$ that have less H I gas relative to their stellar content. These galaxies have more completely converted their gas into stars, endowing them with older stellar populations and redder optical-infrared colors.

To further investigate this additional correlation, in Figure 2 we plot the horizontal deviation of galaxies from the fitted line in panel (d) of Figure 1 versus the $C_{21 W 2}$ colors. In this figure, each black point represents a galaxy, and red points show the average position of galaxies in successive $C_{21 W 2}$ bins. Galaxies with more positive $C_{21 W 2}$ values deviate toward redder $\bar{r}-\bar{W} 2$ values. There are few spirals with $C_{21 W 2}>4$ mag, but it appears that the trend of deviations from the fiducial line levels off around $\bar{r}-\bar{W} 2 \sim 3$. 


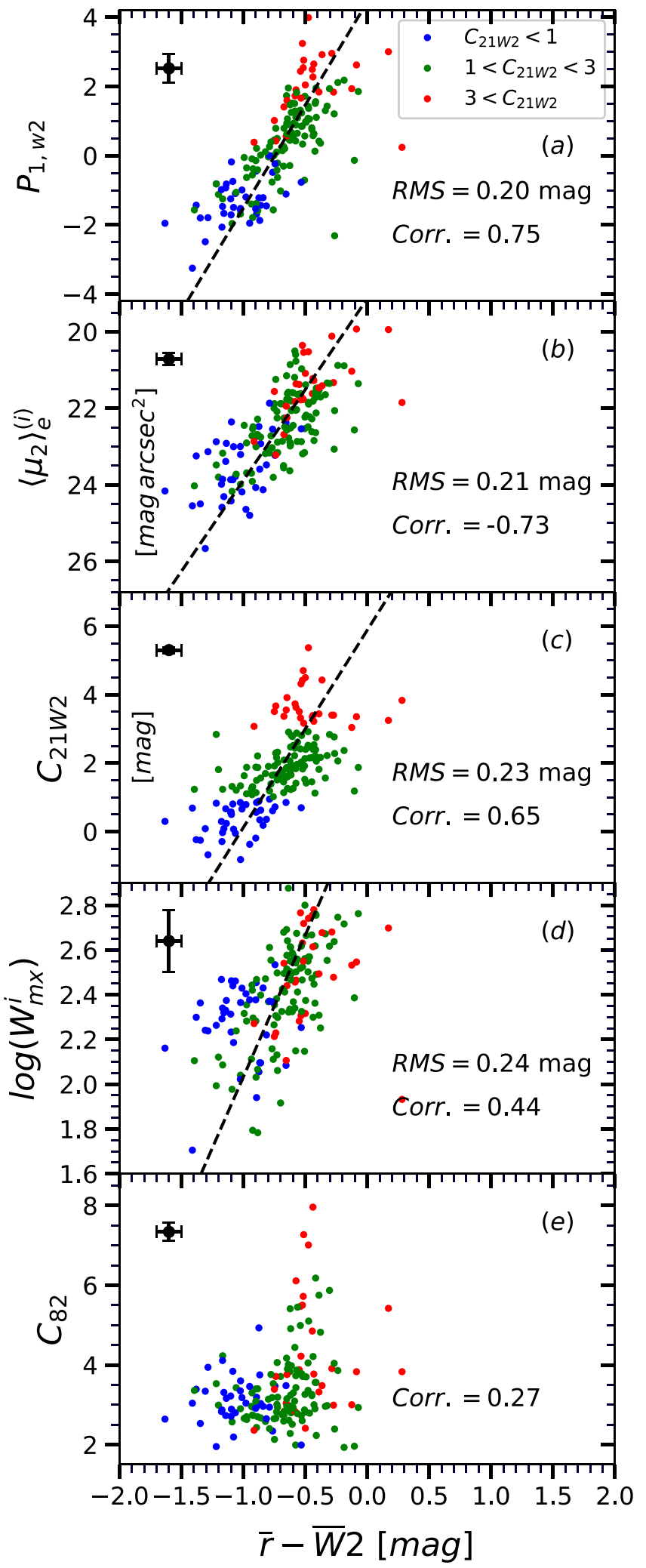

Figure 1. $\bar{r}-\bar{W} 2$ vs. distance-independent observables for galaxies more faceon than $45^{\circ}$. Dashed lines represent the best linear fit with minimizing residuals along the horizontal axis. Each point represents a galaxy with blue, green, and red colors corresponding to $C_{21 W 2}<1,1<C_{21 W 2}<3$, and $C_{21 W 2}>3$, respectively. In each panel, Corr. is the correlation factor for plotted parameters.

Panel (c) of Figure 1 shows the reasonably strong correlation between $\bar{r}-\bar{W} 2$ and $C_{21 W 2}$ colors. The larger correlation coefficient of 0.65 , compared to that of panel (d), 0.44,

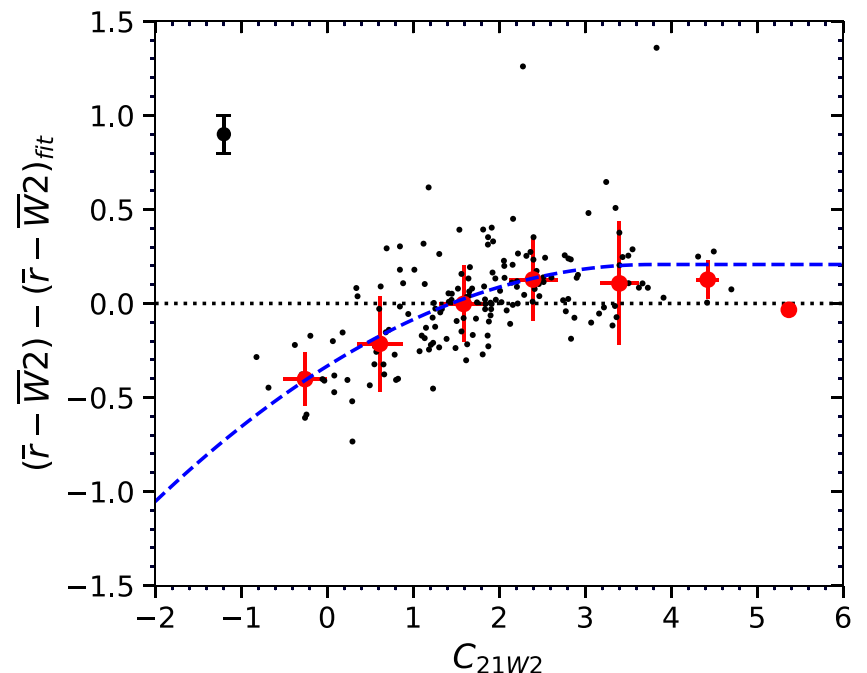

Figure 2. Deviation of $\bar{r}-\bar{W} 2$ colors from the best-fitted line in panel (d) of Figure 10 vs. the HI-IR flux pseudocolor parameter, $C_{21 W 2}$. Each black point represents an individual galaxy. Red points show the median position of black points within $C_{21 W_{2}}$ bins each with the size of $1 \mathrm{mag}$. The error bars on the red points show the $1 \sigma$ scatter of the black points within each bin. The black error bar in the upper left corner shows the typical error bar of the black points. The blue dashed curve displays the best-fitted function to the black points. This function has a quadratic form on the left side and continues as a flat horizontal line after it reaches its maximum. The fitted quadratic function deviates from the null value as $-0.038 C_{21 W 2}^{2}+0.285 C_{21 W 2}-0.333$.

suggests that $C_{21 W 2}$ carries greater information about the optical-infrared parameter. There is a similar correlation with deviations from the fitted line in panel (c) to that with the $\log \left(W_{m x}^{i}\right)$ parameter in panel (d).

The correlation between the effective surface brightness, $\left\langle\mu_{2}\right\rangle_{e}^{(i)}$, measured in $\bar{W} 2$ band and $\bar{r}-\bar{W} 2$ is displayed in the panel (b) of Figure 1. Surprisingly, this correlation is even tighter than those presented in panels (c) and (d).

In conclusion, larger, more intrinsically luminous galaxies have redder stellar population in general, and they tend to have higher surface brightness and lesser fractions of $\mathrm{HI}$ to stellar mass.

\subsection{Distance-independent Principal Components}

In Section 3.1, we presented Figure 2 as an example to show how using the additional correlations that exist between different parameters help to better understand the fiducial colors of face-on spirals. In fact, all the discussed observable features in Section 3.1 are correlated with one another. The left panel of Figure 3 shows the correlation matrix of all features for spirals more face-on than $45^{\circ}$. This matrix holds all the correlation coefficients. The correlation of the color parameter, $\bar{r}-\bar{W} 2$, with other features was presented in detail in Figures 1 and 2. One of the strongest correlations exists between $C_{21 W 2}$ and $\left\langle\mu_{2}\right\rangle_{e}^{(i)}$ with the factor of -0.67 . We find that $\log \left(W_{m x}^{i}\right)$ is mildly correlated to the other features. According to Figure $3, C_{82}$ and $\log \left(W_{m x}^{i}\right)$ have similar behaviors in terms of correlation factors; however, $C_{82}$ has the smallest correlation with the optical-infrared color term. Repeating the same analysis with and without the concentration parameter does not significantly change the outcomes. As such, we decided to remove the concentration parameter from our analysis owing to its small correlations and to avoid distracting our model by incorporating a substantially subdominant feature. 

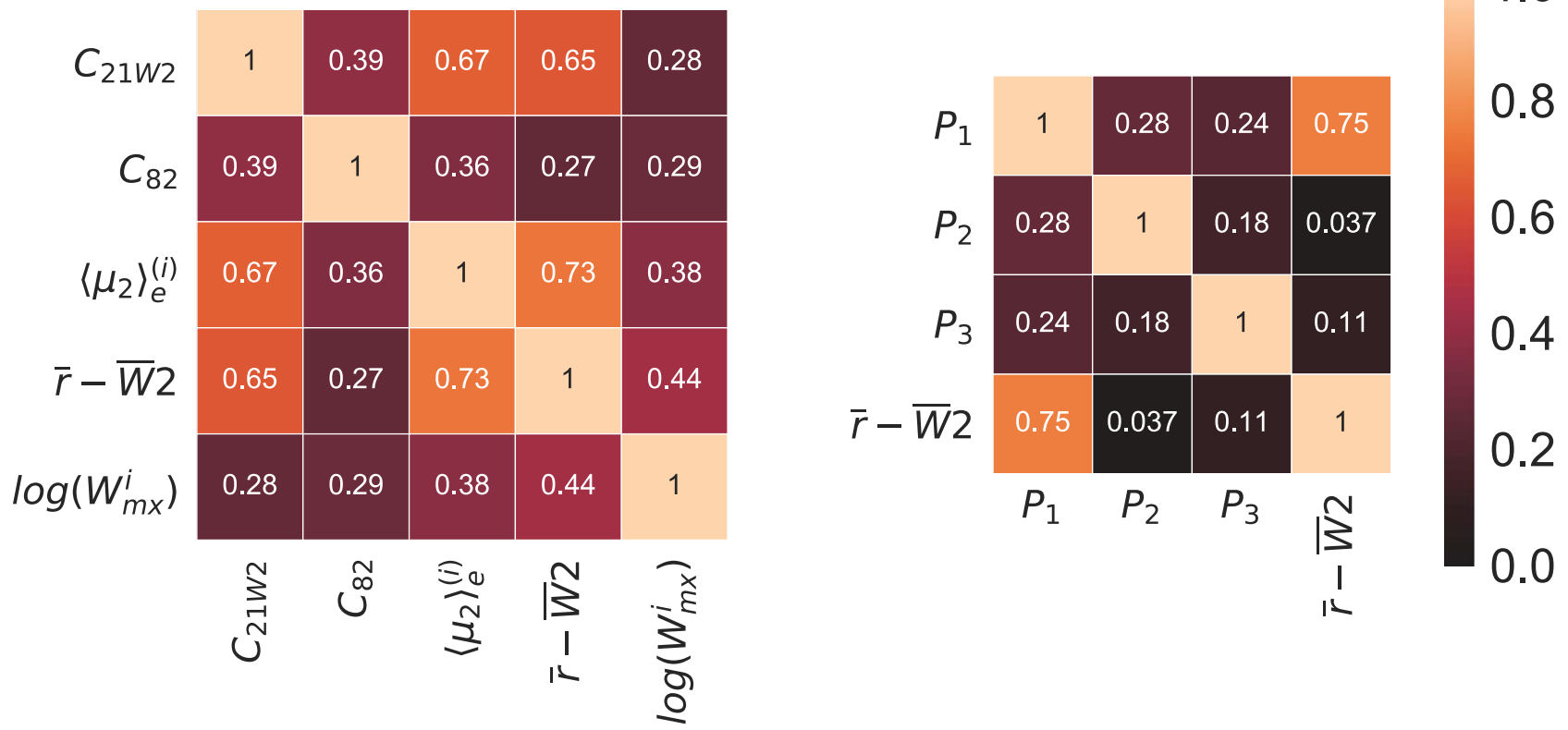

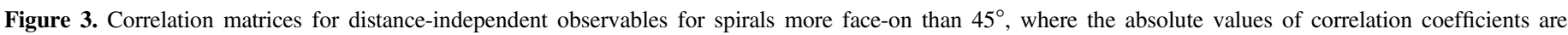
reported.

Due to the mutual correlation of the discussed observable features, and in order to only use the genuine information of each parameter, we perform a principal component analysis (PCA) using $\log \left(W_{m x}^{i}\right), C_{21 W 2}$, and $\left\langle\mu_{j}\right\rangle_{e}^{(i)}$ parameters. PCA outputs an orthogonal transformation that linearly converts the correlated features to uncorrelated principal components. Using a smaller number of principal components compared to the original number of features decreases the sensitivity of results to the uncertainty of measured features by taking advantage of the correlation between features. In fact, one can reduce the noise level of the measured parameters through reducing the number of dimensions by mapping from the input physical features to a space generated by the first few significant principal components followed by an inverse transformation back to the physical space.

Principal components can be derived by linearly combining the observable features following the form

$$
P_{m}=\sum_{i=1}^{3} p_{m, i} X_{i},
$$

where $p_{m, i}$ are weight numbers and $X_{i}$ is the standardized form of a feature $x_{i}$ derived using $X_{i}=\left(x_{i}-u_{i}\right) / \sigma_{i}$, with $u_{i}$ and $\sigma_{i}$ being the mean and standard deviation of the corresponding feature for all sample galaxies with inclinations larger than $45^{\circ}$. These features are

$$
\left\{\begin{array}{l}
x_{1}=\log \left(W_{m x}^{i}\right) \\
x_{2}=C_{21 W j} \\
x_{3}=\left\langle\mu_{j}\right)_{e}^{(i)}
\end{array}\right.
$$

In Equation (6), $\log \left(W_{m x}^{i}\right)$ and $\left\langle\mu_{j}\right\rangle_{e}^{(i)}$ are already corrected for the effect of inclination, and $C_{21 W j}$ is free of the inclinationdependent dust obscuration. Accordingly, for the sake of accuracy, in this part of our analysis we draw our attention to 2014 galaxies in our sample with inclinations larger than $45^{\circ}$ where we have measured their inclinations and used this
Table 2

Principal Components and Standardization Factors Defined in Equations (5) and (6) Using W2-band Photometric Data

\begin{tabular}{lrcrcrr}
\hline \hline$W 2$ & \multicolumn{1}{c}{$p_{1}$} & $p_{2}$ & \multicolumn{1}{c}{$p_{3}$} & Variance Ratio & $u$ & $\sigma$ \\
\hline$P_{1}$ & 0.524 & 0.601 & -0.603 & 0.70 & 2.47 & 0.18 \\
$P_{2}$ & -0.851 & 0.384 & -0.358 & 0.19 & 1.63 & 1.15 \\
$P_{3}$ & 0.016 & 0.701 & 0.713 & 0.11 & 23.35 & 1.38 \\
\hline
\end{tabular}

Table 3

Same as Table 2, but for $W 1$ Band

\begin{tabular}{lrrrcrr}
\hline \hline$W 1$ & \multicolumn{1}{c}{$p_{1}$} & $p_{2}$ & \multicolumn{1}{c}{$p_{3}$} & Variance Ratio & $u$ & $\sigma$ \\
\hline$P_{1}$ & 0.533 & 0.595 & -0.602 & 0.71 & 2.47 & 0.18 \\
$P_{2}$ & -0.844 & 0.420 & -0.332 & 0.18 & 2.11 & 1.17 \\
$P_{3}$ & 0.055 & 0.685 & 0.726 & 0.11 & 22.83 & 1.37 \\
\hline
\end{tabular}

information to calculate the inclination-corrected parameters. The principal component coefficients defined in Equations (5) and (6), together with the normalization factors, $u_{i}$ and $\sigma_{i}$, are presented in Tables 2 and 3. One of the properties of principal components is that the main principal component, $P_{1}$, has the largest variance compared to other components and the input features, and therefore it holds the most information on the scatter of data points. The variance ratio of each principal component is defined as the ratio of its variance by the sum of variances of all individual principal components. The variance ratios are often used to evaluate the significance of principal components. As presented in both Tables 2 and 3, $P_{1}$ carries about $70 \%$ of the information on the scatter in the feature space defined in Equation (6).

We evaluate the performance of the derived principal components for the nearly face-on spirals, where the effect of inclination-dependent dust attenuation on their optical-infrared colors is less significant. In the right panel of Figure 3, the correlation matrix of principal components and $\bar{r}-\bar{W} 2$ color of nearly face-on galaxies is presented. As expected, principal 


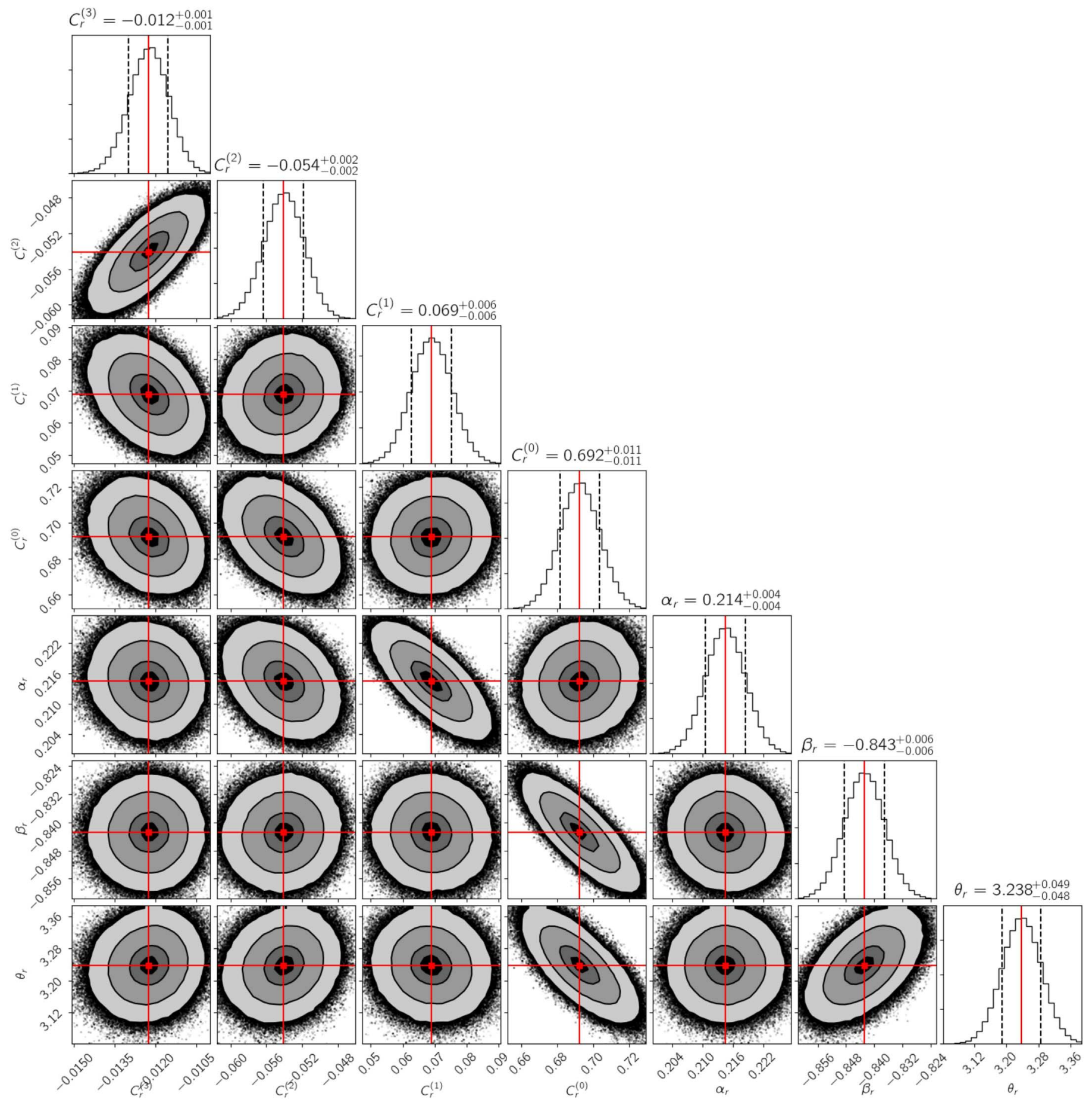

Figure 4. Posterior distribution of the optimized parameters to estimate $\bar{r}-\bar{W} 2=\gamma_{r, W 2}^{(o)}+A_{r, W 2}^{(i)}$. Contours represent $\sigma / 2, \sigma, 3 \sigma / 2$, and $2 \sigma$ levels of the $2 \mathrm{D}$ distributions, and they enclose $12 \%, 39 \%, 68 \%$, and $86 \%$ of the distributed points, respectively. To facilitate the calculations, we set $q_{r}^{2}=10^{-\theta_{r}}$.

components are minimally correlated with one another. Considering both panels of Figure 3, the largest correlation exists between $\bar{r}-\bar{W} 2$ and the main components, $P_{1}$, with the correlation factor of 0.75 . Consistently, panel (a) of Figure 1 also shows how using $P_{1}$ reduces the scatter and establishes a tighter correlation that can serve as a basis for a fiducial relationship.

To summarize, we found that we can replace all correlated parameters in our problem with the first principal component, $P_{1}$, a linear combination in roughly equal parts of line width, ratio of $\mathrm{H} \mathrm{I}$ to old stars, and surface brightness

$$
\begin{aligned}
P_{1, W 2}= & 0.524\left(\log W_{m x}^{i}-2.47\right) / 0.18 \\
& +0.601\left(C_{21 W 2}-1.63\right) / 1.15 \\
& -0.603\left(\left\langle\mu_{2}\right\rangle_{e}^{(i)}-23.35\right) / 1.38
\end{aligned}
$$

that encodes $\sim 70 \%$ of the information on the scatter of data points in the feature space. It is also easier to use only one principal component compared to several features to describe the scatter and general linear trends we observe in Figures 1 

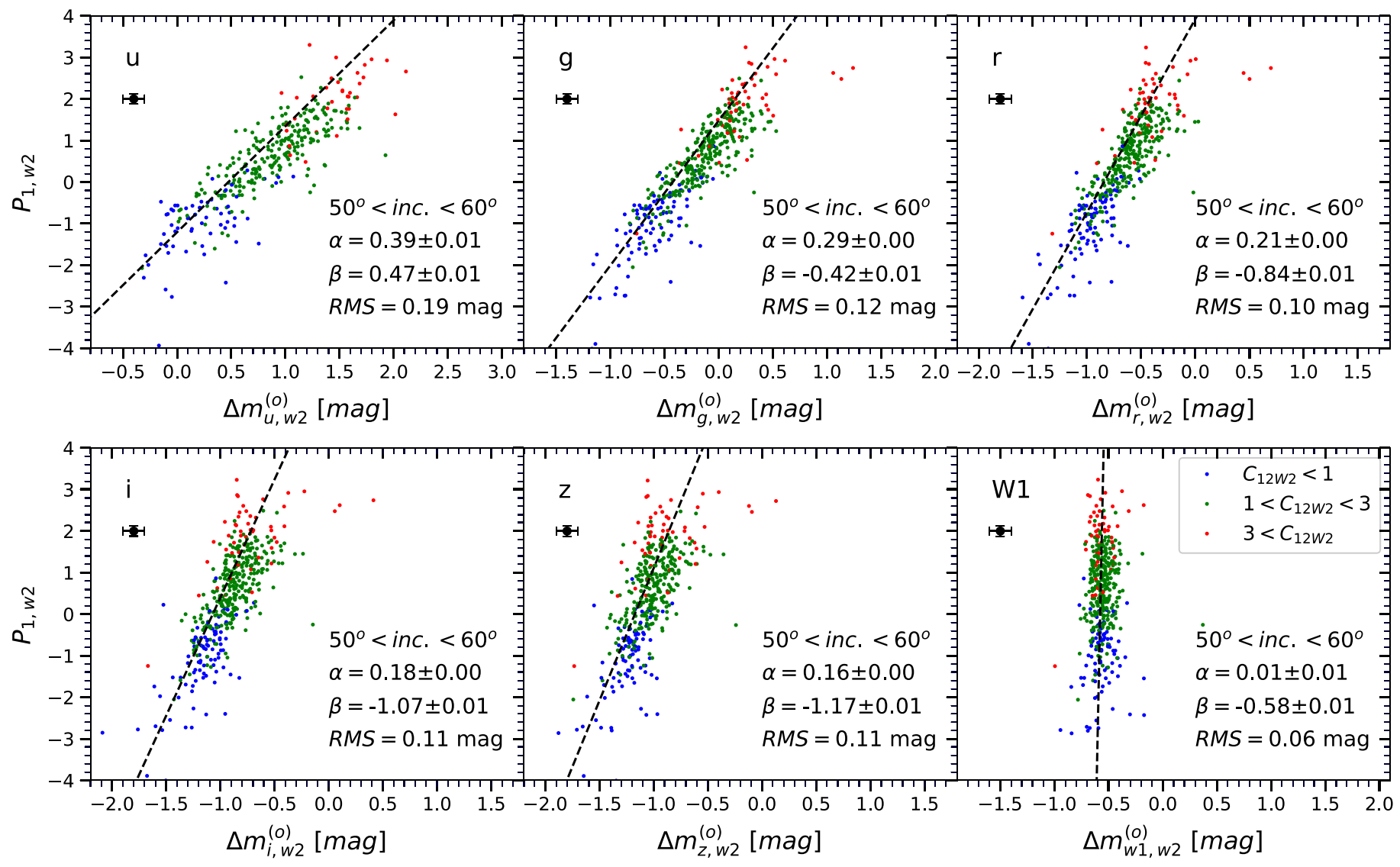

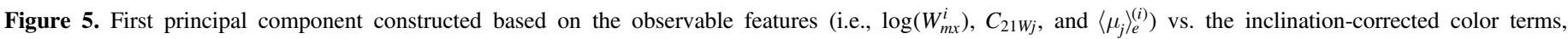

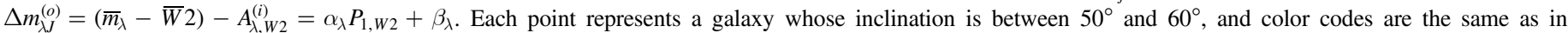

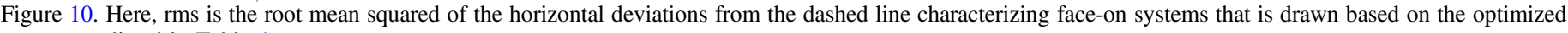
parameters listed in Table 4.

and 2. In addition, any further analysis based on $P_{1}$ is more robust and less prone to outliers and uncertainties in the measured parameters.

The principal components that are alternatively derived based on $W 1$ and $W 2$ fluxes are subject to different levels of dust obscuration and therefore $P_{1, W 1} \neq P_{1, W 2}$. In Appendix C, we compare these principal components and present a procedure to avoid the inclination-dependent biases introduced by using $P_{1, W 1}$.

\section{Method}

In Section 3, we studied the fiducial relation between the optical-infrared color of face-on spirals and their other properties where their luminosities are not influenced by their orientation relative to observer. This study led us to choose the most significant inclination-independent/corrected parameters for the analysis of our entire sample of spirals. Finally, the PCA of these observable features in infrared and $21 \mathrm{~cm}$ wavelength provided us with a novel parameter, $P_{1}$, which can serve as a proxy for the type of spirals by linearly combining their luminosity (or size), surface brightness, and the fraction of $\mathrm{H} \mathrm{I}$ gas to stellar content.

In this section and an appendix, we take two different approaches to empirically model the inclination-dependent attenuation in spiral galaxies. In Section 4.1, considering the studied fiducial relationship, we build a parametric model for the dust attenuation in spirals. In Section 4.2 we sample the posterior distribution of our model parameters using the Markov chain Monte Carlo (MCMC) method. In addition, we tackle the same problem with a nonparametric model based on a Gaussian process (GP) formalism in Appendix A.

\subsection{Parametric Model}

Intuitively, the optical-infrared color of a spiral can be modeled as follows by combining its face-on fiducial color derived from its properties and an extra term responsible for any alteration due to its spatial orientation:

$$
\bar{m}_{\lambda}-\bar{W} j=\Delta m_{\lambda J}^{(o)}+A_{\lambda J}^{(i)},
$$

where $\Delta m_{\lambda J}^{(o)}$ is the fiducial color of a galaxy seen face-on. The effect of inclination angle would be then encoded in $A_{\lambda J}^{(i)}$, which depends on the galaxy properties and its deviation from faceon. The linear fiducial relation we found in Section 3.1 that relates the main principal component of a spiral galaxy and its face-on color suggests the following linear relation:

$$
\Delta m_{\lambda J}^{(o)}=\alpha_{\lambda J} P_{1, J}+\beta_{\lambda J}
$$

where $P_{1 J}$ is the first principal component derived using Equation (5) based on the $\bar{W} j$-band photometry. To be consistent with the standard empirical formalism adopted in the earlier study by Tully et al. (1998), we assume that $A_{\lambda J}^{(i)}$ is 


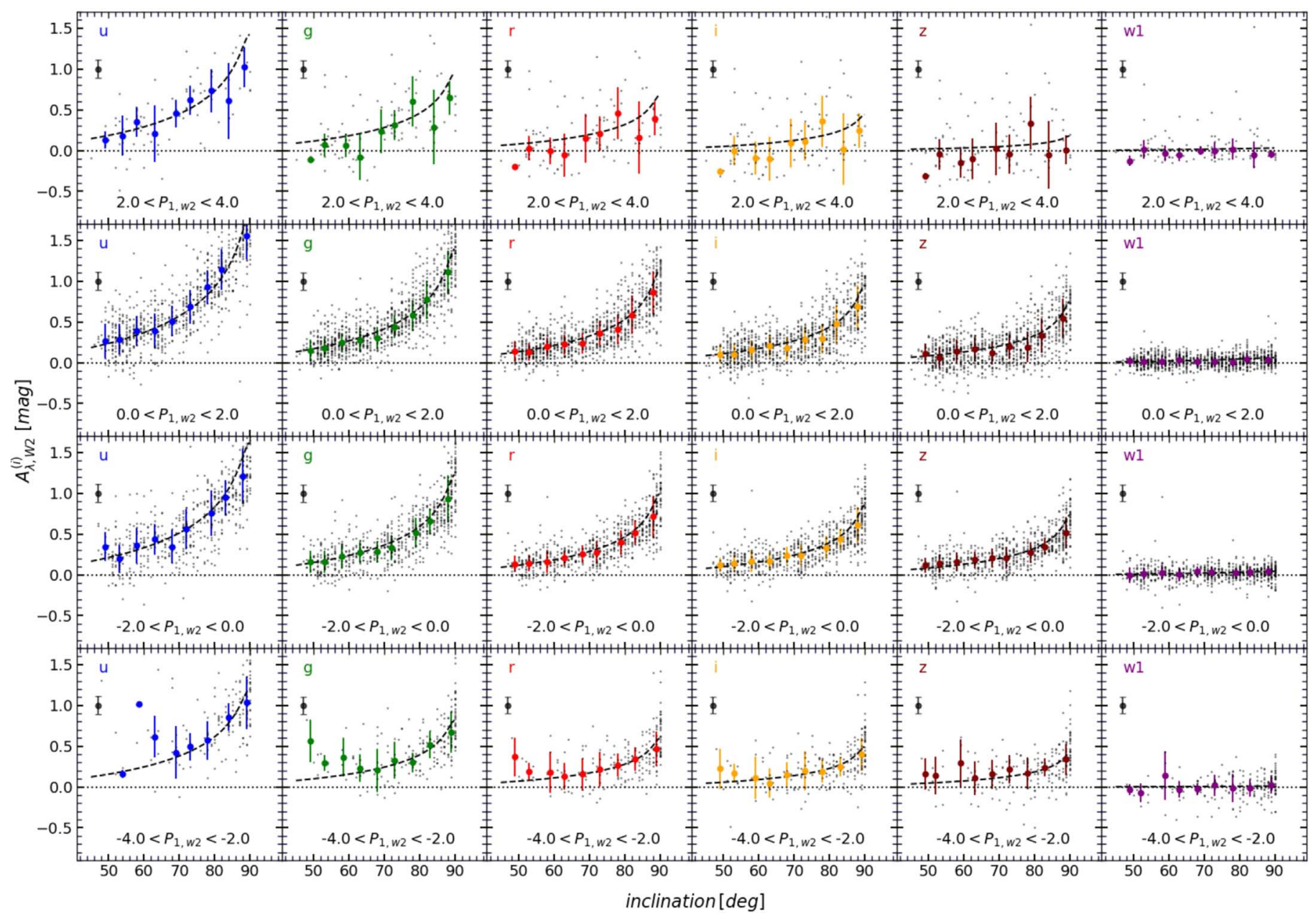

Figure 6. Deviation of galaxies from the fiducial relation, Equation (9), due to their inclination, $A_{\lambda, W 2}^{(i)}$, vs. their inclination for different intervals of $P_{1, W 2}$. Each gray dot represents an individual galaxy, and color points are the average of data points in $5^{\circ}$ inclination bins, with the error bars corresponding to the $1 \sigma$ scatter of data points. The black error barred black point in the upper left corner of each panel displays the median $1 \sigma$ uncertainty on the measured $A_{\lambda, W 2}^{(i)}$ for the displayed spirals. Black dashed curves show our parametric model for the average main principal component of contributing galaxies in each panel.

separable and has the form

$$
A_{\lambda J}^{(i)}=\gamma_{\lambda J} \mathcal{F}_{\lambda}(i)
$$

where $\gamma_{\lambda J}$ is a function of the main principal component and $\mathcal{F}$ depends on the galaxy inclination. We adopt $\mathcal{F}$ to be related to inclination $i$, through

$$
\mathcal{F}_{\lambda}(i)=\log \left[\cos ^{2}(i)+q_{\lambda}^{2} \sin ^{2}(i)\right]^{-1 / 2},
$$

where $i$ is the inclination angle from face-on. $\mathcal{F}_{\lambda}$ is zero for face-on galaxies and increases with inclination to capture the dust attenuation effect along the line-of-sight path length. This relation is very similar to the relation that uses the axial ratio of the photometry aperture, $b / a$, with the form of $\mathcal{F}=\log (a / b)$ (de Vaucouleurs et al. 1991; Bottinelli et al. 1995; Tully et al. 1998), where axial ratio is connected to inclination through $\cos ^{2} i=\left[(b / a)^{2}-q_{o}^{2}\right] /\left(1-q_{o}^{2}\right)$, with $q_{o}$ being the edge-on ellipticity of the spiral disk modeled as a prolate ellipsoid. In our model, $q_{\lambda}$ has a similarity to $q_{o}$; however, it might be interpreted differently. One could think of $q_{\lambda}$ as a wavelengthdependent hyperparameter that, however, does not have any direct geometric meaning. For an edge-on spiral $\left(i=90^{\circ}\right) q_{\lambda}$ sets the peak of $\mathcal{F}_{\lambda}$ and defines the sensitivity of $\mathcal{F}_{\lambda}$ to the galaxy orientation in different wavebands.

Exploring different functional forms, we adopt a third-degree polynomial to model the dependency of $\gamma_{\lambda J}$ on the main principal component of spirals, $P_{1, J}$, that encapsulates the necessary information on galaxy properties. We parameterized this relation as

$$
\gamma_{\lambda J}=\sum_{n=0}^{3} C_{\lambda J}^{(n)} P_{1, J}^{n},
$$

where $C_{\lambda J}^{(n)}$ are the constant coefficients of the adopted polynomial function and $n$ is the degree of each individual term.

\subsection{Optimization}

To find the best parameters of the model introduced in Section 4.1, we follow a Bayesian approach. The objective is to find the posterior probability distribution $\mathcal{P}(\Theta \mid \mathcal{D})$, where $\Theta$ is the vector of all model parameters (i.e., $\alpha_{\lambda J}, \beta_{\lambda J}, C_{\lambda J}^{(n)}$, and $q_{\lambda}$ ). $\mathcal{D}$ holds the observed data, i.e., $P_{1}$ and inclination, for all spirals with inclinations greater than $45^{\circ}$. Conditional probability law indicates that $\mathcal{P}(\Theta \mid \mathcal{D}) \propto \mathcal{P}(\mathcal{D} \mid \Theta) \mathcal{P}(\Theta)$. 


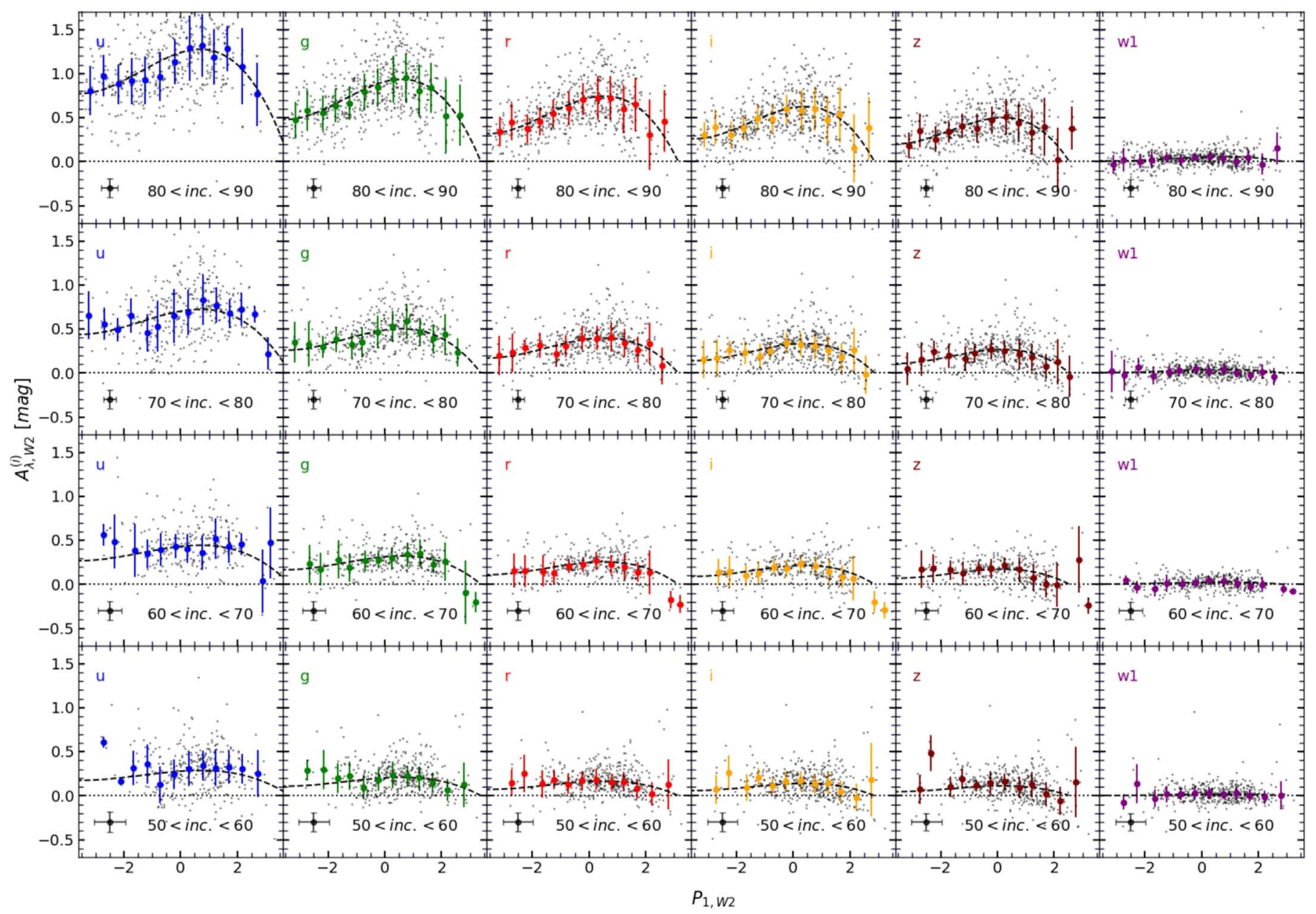

Figure 7. Reddening parameter $A_{\lambda, W 2}^{(i)}$ vs. principal component parameter $P_{1, W 2}$ for different inclination ranges. Color points represent the average of data points in horizontal bins with the size of 0.5 .

The lack of any prior knowledge on the distribution of the model parameters leads us to set $\mathcal{P}(\Theta)=1$. This assumption simplifies our problem, which could be solved adopting any optimization technique to maximize the likelihood function, defined as $\mathcal{L}=\mathcal{P}(\mathcal{D} \mid \Theta)$. Nevertheless, we take advantage of the MCMC method to explore the parameter space and to sample the posterior distribution by incorporating all uncertainties of the observed data. The independence of each data point associated with a galaxy from the measured parameters of other galaxies, as well as the Gaussian nature of uncertainties, results in the following likelihood function:

$$
\mathcal{L}=\prod_{n=1}^{N} \frac{1}{\sqrt{2 \pi \sigma_{n}^{2}}} \exp \left(\frac{\mathcal{D}_{n}-\mathcal{M}_{n}(\Theta)}{\sigma_{n}}\right)^{2},
$$

where $n$ is the galaxy index and $N$ is the total number of sample galaxies. For each galaxy $\mathcal{D}_{n}=\left(\bar{m}_{\lambda}-\bar{W} j\right)_{n}$ is calculated using the observed values of the optical and infrared magnitudes, $\mathcal{M}_{n}$ is the output of the parametric model described by Equation (8), and $\sigma_{n}$ is the uncertainty of the $\mathcal{D}_{n}-\mathcal{M}_{n}(\Theta)$ parameter that acts as a weight factor that penalizes a model based on its prediction from real measurements and is calculated using $\sigma_{n}^{2}=\sigma_{D_{n}}^{2}+\sigma_{\mathcal{M}_{n}}^{2}$. One can obtain $\sigma_{D_{n}}$ by adding the uncertainties of the measured magnitudes in quadrature. For any model with given parameters, $\Theta$, the model uncertainty $\sigma_{\mathcal{M}}$ is calculated through propagating uncertainties on observable features, i.e., $\sigma_{P_{1}}$ and $\sigma_{i}$.

We use the Python package emcee (Foreman-Mackey et al. 2013) to sample the posterior distribution. To accelerate calculation by utilizing fast linear algebra routines, emcee uses the logarithm of the posterior likelihood

$$
\log \mathcal{L}(\boldsymbol{r})=-\frac{1}{2} \boldsymbol{r}^{T} \Sigma^{-1} \boldsymbol{r}-\frac{1}{2} \log |\Sigma|-\frac{N}{2} \log (2 \pi)
$$

where $\boldsymbol{r}$ is the $N \times 1$ data-model residual vector whose elements are $r_{n}=\mathcal{D}_{n}-\mathcal{M}_{n}(\Theta)$ and $\Sigma$ is the $N \times N$ diagonal covariance matrix where $\Sigma_{n, n}=\sigma_{n}^{2}$. Adopting this likelihood function, for each photometry band we generate 200 chains each with the length of 20,000 samples. To expand the size of explored regions in the parameter space, each chain is initialized randomly. We observed that after almost 500 burning steps, each chain converges and follows the Markov statistics. To be more conservative, we removed the first 2000 steps of each MCMC sample and combined all 200 walkers to construct the posterior distribution of model parameters. Figure 4 illustrates the corner plot for the distribution of the sampled distribution of parameters to model $\bar{r}-\bar{W} 2$. In this diagram, the top panel of each column shows the 1D distribution of the corresponding sampled parameter, displayed 

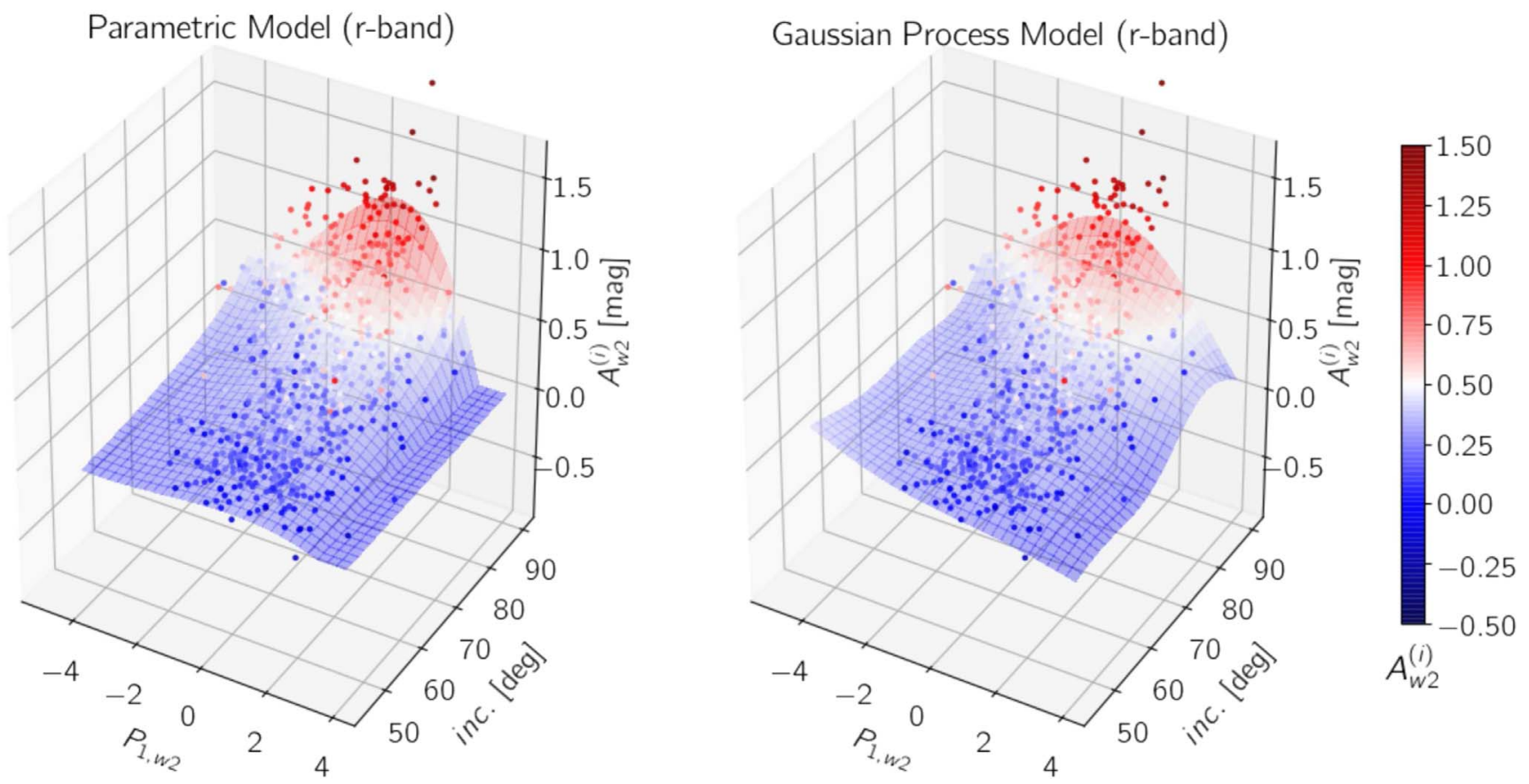

Figure 8. The $r$-band inclination-dependent dust obscuration in spiral galaxies, $A_{r, W 2}^{(i)}$, as a function of their spatial inclination relative to the observer line of sight and their main principal component, $P_{1}$ (Equation (5)). Left: best-fitted parametric model formulated by Equation (10) and parameters in Table 4. Right: nonparametric model based on a GP formalism described in Appendix A. Each meshed surface shows the corresponding model, whereas the colored points illustrate the location of galaxies in this space. To avoid confusion, only 700 data points are chosen randomly and overplotted.

with the median values (red solid line) and the lower/upper bounds corresponding to 16 th/84th percentiles (black dashed line). Horizontal and vertical red lines show the location of the median values, which we adopt as the optimum values of our model parameters. The shape of the posterior distribution looks almost the same as in Figure 4 for all optical bands.

Figure 5 illustrates how spirals in the inclination range between $50^{\circ}$ and $60^{\circ}$ are distributed relative to our model outcome for the linear fiducial relation between face-on color, $\Delta m_{\lambda J}^{(o)}$, and main principal component, $P_{1, W 2}$, as defined by Equation (8). Despite the similarity, we draw the reader's attention to the difference between the presented fiducial lines in this diagram and that presented in Section 3.1.

In Figure 1, we only used face-on galaxies with no measurement for their inclinations, where we set all inclinations to $40^{\circ}$ to explore the color dependency of face-on spirals on different parameters, by fitting the fiducial line using the plotted scattered points. However, in Figure 5 we take the optimized parameters of our model (Table 4), which are found based on all galaxies with inclinations $>45^{\circ}$ to overplot the fiducial relation on the scattered data points of spirals with inclinations that range between $50^{\circ}$ and $60^{\circ}$.

Figure 6 shows different cross sections of our resulting models for $A_{\lambda, W 2}^{(i)}$ at different wavelengths versus inclination for different ranges of $P_{1, W 2}$. In these plots each gray dot represents a galaxy whose $A_{\lambda, W 2}^{(i)}$ value is calculated following

$$
A_{\lambda, W 2}^{(i)}=\left(\bar{m}_{\lambda}-\bar{W} 2\right)-\left(\alpha_{\lambda} P_{1, W 2}+\beta_{\lambda}\right),
$$

where $\alpha_{\lambda}$ and $\beta_{\lambda}$ are the model parameters for the linear fiducial relation of face-on colors taken from Table 4. While $\alpha_{\lambda}$ and $\beta_{\lambda}$ are not purely observable quantities, they could be separately obtained from an independent analysis of fiducial linear relation of face-on spirals. Our roughly estimated $\alpha_{\lambda}$ and

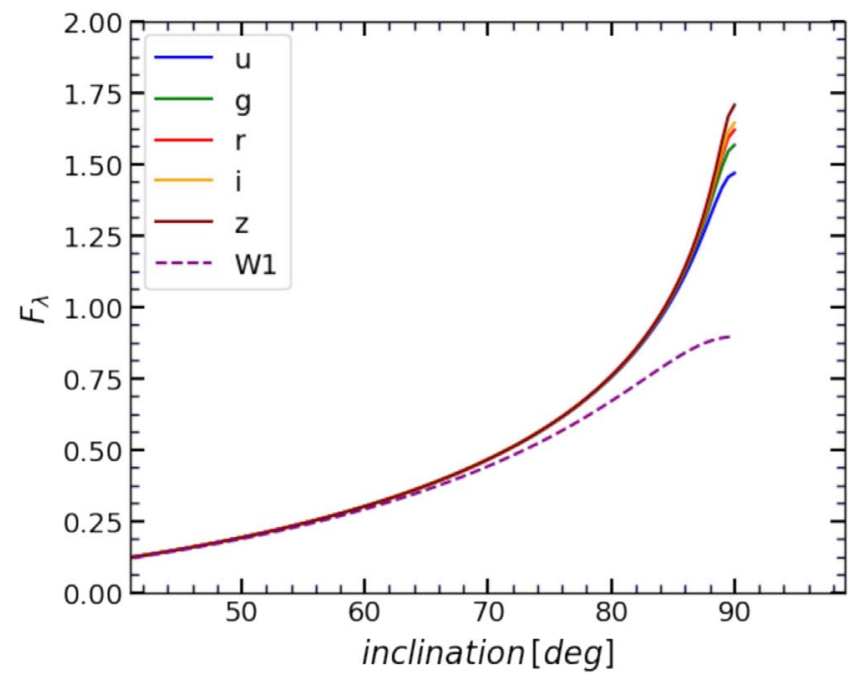

Figure 9. Inclination-dependent component of dust attenuation, $\mathcal{F}_{\lambda}(i)$, as defined in Equation (11), with separate $q_{\lambda}$ values taken from Table 4.

$\beta_{\lambda}$ values for close-to-face-on galaxies in Section 3.1 and what we get from our precise optimization of our parametric model (Table 4) point us to almost the same fiducial trends. As defined in Equation (15), $A_{\lambda, W 2}^{(i)}$ for each galaxy represents its color deviation from the fiducial relation of face-on spirals attributable to the inclination-dependent dust attenuation.

In Figure 6, to better see the general trend of the data points, the color points show the mean of $A_{\lambda, W 2}^{(i)}$ for galaxies in the inclinations bins of size $5^{\circ}$, where the vertical error bars display $1 \sigma$ standard deviation of the scatter along the attenuation parameter. In each panel, the black dashed curve is the result of our model, as we use the median of the $P_{1, W 2}$ value for all galaxies in that panel. The parameter $\mathcal{F}_{\lambda}(i)$ with fixed $q_{\lambda}$ 


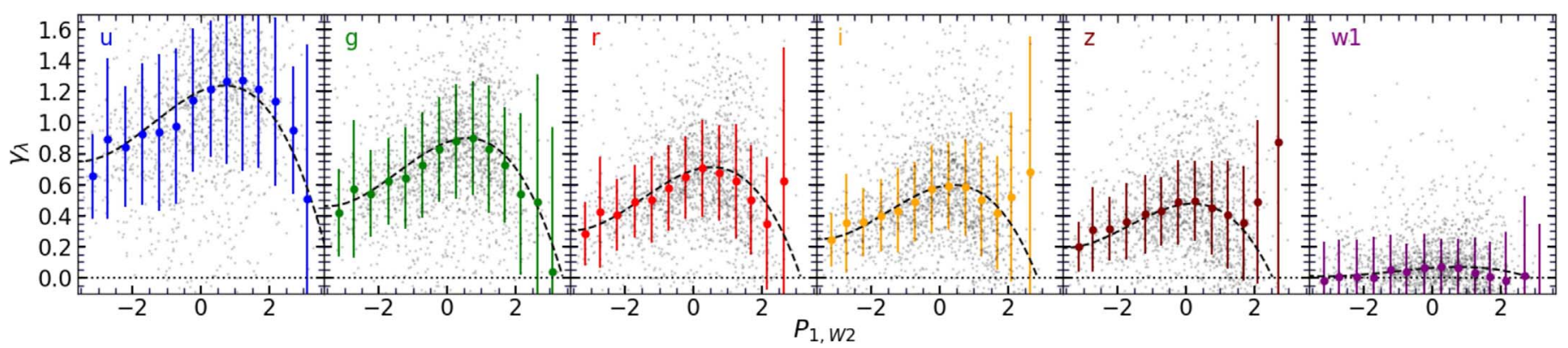

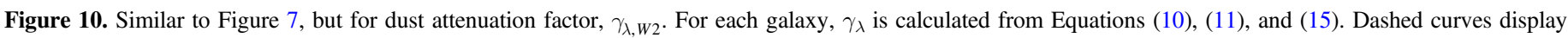
Equation (12) with the optimized parameters taken from Table 4.

Table 4

Optimized Parameters of the Parametric Model to Describe $\bar{m}_{\lambda}-\bar{W} 2$ Defined by Equation (8)

\begin{tabular}{|c|c|c|c|c|c|c|c|}
\hline$\lambda$ & $C_{\lambda}^{(3)}$ & $C_{\lambda}^{(2)}$ & $C_{\lambda}^{(1)}$ & $C_{\lambda}^{(0)}$ & $\alpha_{\lambda}$ & $\beta_{\lambda}$ & $\theta_{\lambda}=-2 \log \left(q_{\lambda}\right)$ \\
\hline$u$ & $-0.013 \pm 0.001$ & $-0.055 \pm 0.003$ & $0.096 \pm 0.009$ & $1.202 \pm 0.018$ & $0.392 \pm 0.006$ & $0.473 \pm 0.008$ & $2.935 \pm 0.041$ \\
\hline$g$ & $-0.013 \pm 0.001$ & $-0.056 \pm 0.002$ & $0.081 \pm 0.007$ & $0.873 \pm 0.013$ & $0.287 \pm 0.004$ & $-0.424 \pm 0.006$ & $3.131 \pm 0.042$ \\
\hline$r$ & $-0.012 \pm 0.001$ & $-0.054 \pm 0.002$ & $0.069 \pm 0.006$ & $0.693 \pm 0.011$ & $0.214 \pm 0.004$ & $-0.843 \pm 0.006$ & $3.237 \pm 0.049$ \\
\hline$i$ & $-0.012 \pm 0.001$ & $-0.055 \pm 0.003$ & $0.051 \pm 0.007$ & $0.586 \pm 0.010$ & $0.176 \pm 0.004$ & $-1.071 \pm 0.005$ & $3.286 \pm 0.054$ \\
\hline$z$ & $-0.011 \pm 0.001$ & $-0.055 \pm 0.003$ & $0.023 \pm 0.007$ & $0.474 \pm 0.010$ & $0.157 \pm 0.004$ & $-1.173 \pm 0.005$ & $3.411 \pm 0.063$ \\
\hline$W 1$ & $-0.002 \pm 0.000$ & $-0.007 \pm 0.000$ & $0.015 \pm 0.011$ & $0.062 \pm 0.018$ & $0.008 \pm 0.005$ & $-0.578 \pm 0.006$ & $1.789 \pm 0.068$ \\
\hline
\end{tabular}

controls how attenuation varies with inclination (see Equation (11)). As expected, the effect of dust attenuation decreases toward longer wavelengths, with minimal effect at W1 band.

Similarly, Figure 7 shows the behavior of our model with variance of the principal component against data points in different intervals of inclinations. For a given inclination, $A_{\lambda, W 2}^{(i)}$ is related to the main principal component through a polynomial function defined by Equation (12), with a peak around $P_{1, W 2} \simeq 1$. As expected, for a constant $P_{1, W 2}$ value, the dust attenuation increases as galaxies become more edge-on.

The 3D behavior of our model is seen in Figure 8, where we plot $A_{r, W_{2}}^{(i)}$ as a function of inclination and the main principal component constructed from the $W 2$-band photometry. We set the attenuation to zero where the parametric model goes negative at $P_{1, W 2} \gtrsim 3$.

\subsection{Comparisons with a Semiparametric Model}

In Section 4.1, we constructed a parametric model of the effect of inclination on the fiducial color relations of the faceon galaxies. In this section, we take the GP approach to predict $A_{\lambda J}^{(i)}$ for a set of parameters $P_{1, J}$ and inclination, based on the information provided by the observed data for neighboring sample spirals in the parameter space. GP is a statistical machine learning technique based on a Bayesian approach that generates predictions following the behavior of data (e.g., Rasmussen \& Williams 2006; Gibson et al. 2012; Rezaei et al. 2017). Considering the strong correlations that underlie the fiducial relations for face-on spirals, we continue using Equation (9) with the best-fitted $\alpha_{\lambda J}$ and $\beta_{\lambda J}$ taken from Table 4 followed by a GP formalism to model $A_{\lambda J}^{(i)}$. See Appendix A for the details of our GP algorithm setup.

Figure 8 compares the results of the parametric and GP models. Both models tend to underestimate a flare in the dust attenuation at $\sim 90^{\circ}$, although our parametric model appears to perform better at larger inclinations. In regions with numerous data points, say, $-2<P_{1}<2$, the results of our alternate models are almost the same; however, they differ significantly in less populated regions. The behavior of the parametric model is controlled by the adopted formulation that reasonably captures the physics of the problem, while the GP formalism solely learns from the distribution of data points to make predictions. Thus, one of the main downsides of the GP model in following the trend of data points is that it performs poorly wherever few measurements are available. The differences between parametric and GP models are modest, but we give preference to the parametric model owing to its simplicity.

\section{Dependency of Dust Attenuation on Wavelength}

The inclination-dependent dust attenuation was modeled independently in multiple passbands in Section 4.1. The model follows Equation (10), and the best-fitted parameters are presented in Table 4 . In this equation, $\gamma_{\lambda}$ depends only on the observed properties of galaxies encoded in $P_{1, W 2}$, and $\mathcal{F}_{\lambda}(i)$ is a function of galaxy inclination. Figure 9 displays the behavior of $\mathcal{F}_{\lambda}(i)$ in different passbands. As seen, except for very edge-on spirals, at optical wavelengths $\mathcal{F}_{\lambda}$ is almost the same for all inclination values. We observe that the W1-band curve is shallower compared to those at optical bands, which is consistent with our expectation that dust absorption is less sensitive to inclination in longer wavelengths.

Absorption and inclination can be decoupled to give the physically meaningful dust attenuation factor $\gamma_{\lambda}=A_{\lambda, W 2}^{(i)} /$ $\mathcal{F}_{\lambda}(i)$. In Figure 10, we plot $\gamma_{\lambda}$ as a function of $P_{1, W 2}$ in different wavebands. Each gray dot represents a spiral whose $\gamma_{\lambda}$ value is derived following Equations (10), (11), and (15). Dashed curves plot $\gamma_{\lambda}$ as parameterized in Equation (12) using the optimized parameters presented in Table 4. Consistent with our expectation, dust obscuration is greater in shorter wavebands with a similar dependency on $P_{1, W 2}$ at all frequencies. We can see how dust attenuation in spiral galaxies varies with wavelength. Panels of Figure 11 plot dust attenuation in spirals relative to that in $g$ band. In each panel, $\gamma_{\lambda} / \gamma_{g}$ for each galaxy is calculated by taking the corresponding 


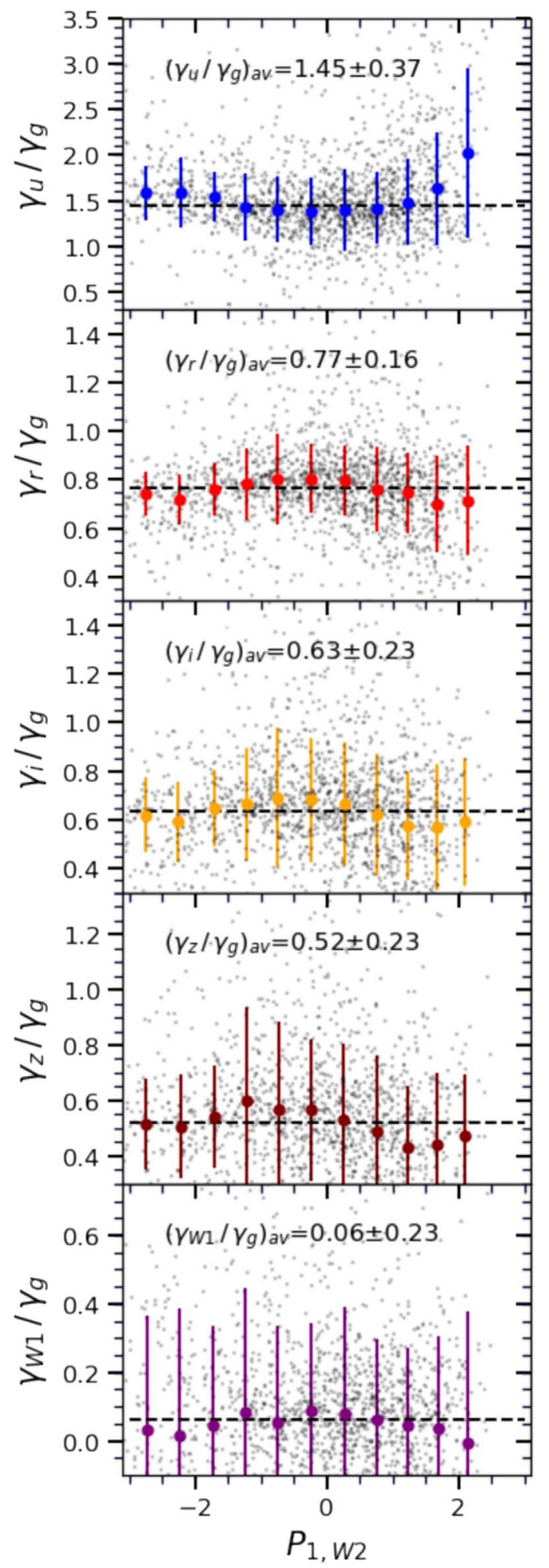

Figure 11. Similar to Figure 10, but for the ratio of dust attenuation in different wavebands. In each panel, the dashed horizontal line shows the average extinction ratio, $\left(\gamma_{\lambda} / \gamma_{g}\right)_{a v}$, of all galaxies represented by gray dots.

$\gamma_{\lambda}$ values from Figure 10. Color points and their error bars show median and $1 \sigma$ scatter of gray dots in bins with the size of 0.5 along $P_{1, W 2}$. In each panel, a dashed horizontal line is drawn at the level of $\left(\gamma_{\lambda} / \gamma_{g}\right)_{a v}$, which is derived by taking the median of all $\gamma_{\lambda} / \gamma_{g}$ fractions for all spirals. The scatter of color points and the size of their error bars do not imply any significant dependency of mean $\gamma_{\lambda} / \gamma_{g}$ value on $P_{1, W 2}$.

Dust extinction, $A_{\lambda}$, is usually normalized by color excess $E(B-V) \equiv A_{B}-A_{V}$,

$$
\kappa_{\lambda}=\frac{A_{\lambda}}{E(B-V)}=\frac{A_{\lambda}}{A_{B}-A_{V}},
$$

where $\kappa_{\lambda}$ is the dust extinction factor. The value of $\kappa_{\lambda}$ in $V$ band for the Milky Way is $R_{V} \equiv \kappa_{V}=A_{V} / E(B-V)=3.1$ (Cardelli et al. 1989). According to this formalism, dust extinction ratios are given as

$$
A_{\lambda} / A_{V}=\kappa_{\lambda} / \kappa_{V}=\kappa_{\lambda} / R_{V}
$$

Adopting $\left(\gamma_{\lambda} / \gamma_{g}\right)_{a v}$ values from Figure 11, we display the wavelength dependency of attenuation ratios, $A_{\lambda} / A_{g} \simeq\left(\gamma_{\lambda} / \gamma_{g}\right)_{a v}$, in Figure 12. As a reference, reddening curves for the Milky Way (Cardelli et al. 1989) and Small Magellanic Cloud (SMC) bar (Gordon et al. 2003) are overplotted.

In Figure 10 of their paper, Gordon et al. (2003) present the reddening laws for several galaxies that suggest a linear dependency on the inverse of wavelengths in optical and infrared ranges. We find the following linear relationship for the average of our measured relative dust attenuation by fitting a straight line that passes through the $g$-band point:

$$
\frac{\gamma_{\lambda}}{\gamma_{g}}=(1.097 \pm 0.060)\left[\frac{\lambda_{g}}{\lambda}-1\right]+1,
$$

where $\lambda_{g}=4886 \AA$ is the central wavelength of the SDSS $g$-band filter. Setting $\lambda_{V}=5500 \AA$, Equation (18) implies $\gamma_{\lambda} / \gamma_{V}=1.194\left(\gamma_{\lambda} / \gamma_{g}\right)$

We note that at longer wavelengths our measured values for attenuation do not fall as rapidly as for the standard Galactic reddening case. This is in agreement with the average dust attenuation curve derived by Salim et al. (2018), where a similar behavior is noticeable. The Galactic reddening curve pertains to the extinction over a range of wavelengths to a fixed source. By contrast, the internal dust obscuration that we measure in galaxies averages over multiple paths to varying depths into a target that depends on wavelength. At longer wavelengths galaxies become less optically thick and thus pass photons from more embedded regions (Han 1992; Tully et al. 1998). Integrating the obscuration over a longer line-of-sight path consequently amplifies the effect of dust attenuation compared to Galactic extinction along a path to a fixed source. Furthermore, extinction involves photons scattered into the line of sight, which can result in significant discrepancy between extinction and attention curves.

In Appendix B, we find an empirical relation between $\gamma_{\lambda}$ and effective surface brightness, $\left\langle\mu_{2}\right\rangle_{e}^{(i)}$, which is useful when $\mathrm{HI}$ $21 \mathrm{~cm}$ data are unavailable.

\section{Summary}

Photons that are produced by the stellar content of a galaxy are absorbed and/or scattered by dust grains and gas molecules in its ISM. Physical properties and chemical composition of dust particles in the ISM of a galaxy depend on different factors like galaxy stellar ages, mass, morphology, metallicity, and formation history. The level of obscuration also depends on the spatial inclination of the galaxy relative to the observer. In galaxies with larger inclinations from face-on, the average 


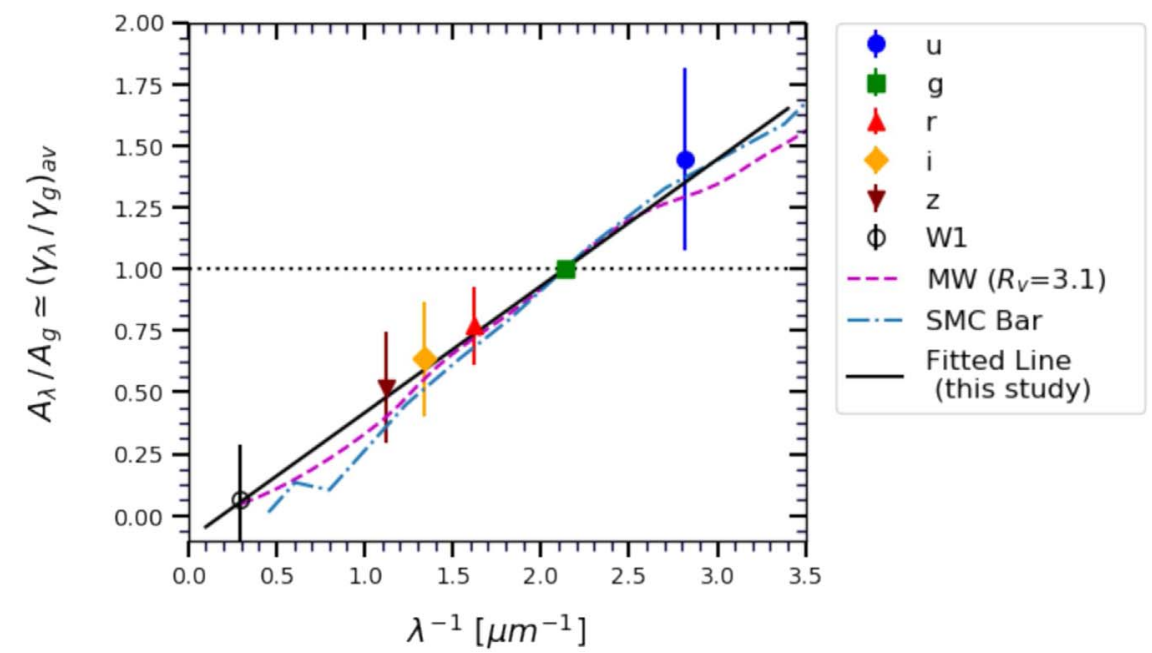

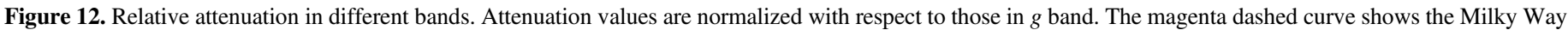

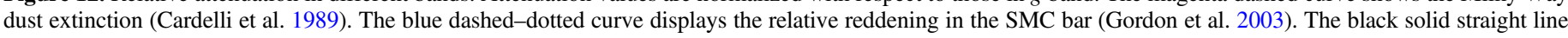
displays the best-fitted linear relation given by Equation (18).

photon travels along a longer line-of-sight path within the host and therefore has a higher chance to be obscured.

The current study is an attempt to model the inclinationdependent dust obscuration in spiral galaxies at multiple optical and infrared wavelengths. To acquire the necessary information to conduct this study, we performed photometry on a sample of 2239 spirals with optical and infrared images provided by SDSS and WISE surveys. Another crucial piece of information is the inclinations of spirals that were carefully measured by visually comparing them to a set of standard spirals with known inclinations.

Empirically, larger galaxies have fractionally smaller $\mathrm{HI}$ components and, by inference, must be more efficient in consuming their $\mathrm{HI}$ gas and forming stars. In the present universe, on average more massive galaxies tend to have older/ redder stellar populations and have relatively less $\mathrm{H}$ I gas. Prior to addressing the dependency of dust attenuation on inclination, we have established a set of inclination-independent fiducial relations at different wavebands to capture this general colorsize trend. We found relationships that describe the opticalinfrared colors of face-on spirals in terms of other observable features that are correlated with their physical properties (Sections 3.1 and 3.2).

Turning to spirals with greater inclinations, we then model the inclination dependency of deviations of optical-infrared colors from the fiducial relations. These departures are attributed to the inclination-dependent effects of dust obscuration on the observables.

In Section 4.1 we established a parametric model to describe attenuation as a function of inclination and other observables. Taking a nonparametric approach in Appendix A, we used the GP formalism to study dust obscurations, and we show that the differences between our two methods are not significant.

Phenomenologically, both dwarf galaxies and galaxies proceeding toward red and dead have negligible optical attenuation. On the other hand, intermediate spiral galaxies can lose well more than half their light to attenuation at blueward bands. Low-mass galaxies can have fractionally large amounts of $\mathrm{HI}$, but this component of interstellar material is optically transparent. Small galaxies have low metal content and have very limited molecular gas reservoirs
(Yates et al. 2012; Bothwell et al. 2016). Dust grains in the ISM are the products of the release of metals due to star formation activity and the late-stage evolution of stars. In the regime more massive than dwarfs that interests us, there is a roughly linear logarithmic relation between dust-to-gas ratio and metallicity (Leroy et al. 2011; Feldmann 2015). Global galaxy metallicity can serve as a proxy of galaxy dust levels (Marassi et al. 2019). Mean metallicity increases with galaxy mass until it saturates and grows only slowly for galaxies more massive than $M_{*} \simeq 10.5 M_{\odot}$ (Tremonti et al. 2004; Zahid et al. 2014).

A generally consistent picture emerges. Proceeding from smaller to larger masses, the metal content of the ISM increases within abundant $\mathrm{H}$ I reservoirs, resulting in increasing attenuation from opaque nebulosity. At some point in this progression to more massive systems, though, the level of metallicity no longer grows significantly, while the $\mathrm{HI}$ content becomes increasingly depleted. Stellar winds or active galactic nuclei are increasingly effective at clearing the galaxy of the ISM (Peeples \& Shankar 2011; Kudritzki et al. 2015).

Our principle component parameter $P_{1, J}$ captures the essence of this interplay. This parameter is a linear combination of three distance-independent observables: (1) the amplitude of galactic rotation, which is a proxy for total galaxy mass, (2) an H I to infrared flux pseudocolor that monitors the relative amount of gas available to form young stars versus the quantity of old stars, and (3) the infrared surface brightness. Dust attenuation grows with $P_{1, J}$ until it reaches a maximum and turns over around $P_{1, J} \simeq 1$. Increasing galaxy mass is a factor causing increasing obscuration, while an increasingly red pseudocolor $C_{21 W 2}$ is a factor in diminishing obscuration. Interestingly, the single parameter that comes closest to capturing the essence of the $P_{1, J}$ correlation with attenuation is the infrared surface brightness.

The differential reddening from SDSS $u$ band to WISE W2 band is only slightly different from the familiar Galactic reddening law with $R_{V}=3.1$. As can be anticipated, mean reddening is slightly greater at longer wavelengths than anticipated by the Galactic reddening law to a fixed source because separate lines of sight penetrate hosts to different depths as a function of wavelength. 
We are pleased to acknowledge the citizen participation to scientific research of undergraduate students at University of Hawaii, members of amateur astronomy clubs in France Planétarium de Vaulx-en-Velin, Association Clair d'étoiles et Brin d'jardin, Société astronomique de Lyon, Club d'astronomie Lyon Ampère, Club d'astronomie des monts du lyonnais, Club d'astronomie de Dijon, and friends who helped us with measuring inclinations of spiral galaxies in our sample.

Support for E.K. and R.B.T. was provided by NASA through grant No. 88NSSC18K0424 from the Space Telescope Science Institute. H.C. acknowledges support from Institut Universitaire de France.

This research has made use of the NASA/IPAC Extragalactic Database, ${ }^{15}$ which is operated by the Jet Propulsion Laboratory, California Institute of Technology, under contract with the National Aeronautics and Space Administration. This research made use of Montage, funded by the National Aeronautics and Space Administrations Earth Science Technology Office, Computational Technologies Project, under Cooperative Agreement No. NCC5-626 between NASA and the California Institute of Technology. The code is maintained by the NASA/IPAC Infrared Science Archive.

\section{Appendix A GP Recipe to Model $A_{\lambda J}^{(i)}$}

Following the GP algorithm, we assume that $N$ observations for $A_{\lambda J}^{(i)}$ are drawn from a prior normal Gaussian distribution, i.e., $A_{\lambda J}^{(i)} \sim \mathcal{N}(0, \mathcal{K})$, where the mean is zero and $\mathcal{K}$ is the $N \times N$ covariance matrix generated using the uncertainty of $A_{\lambda J}^{(i)}$ and the GP kernel function that is chosen based on the expected behavior of data points. Let $\boldsymbol{A}$ be an $N \times 1$ column vector whose elements are $A_{\lambda J}^{(i)}$ measured for $N$ spirals. For any pair of galaxies, the covariance matrix element is written as

$$
\mathcal{K}_{a b}=\sigma_{A_{a}}^{2} \delta_{a b}+\kappa_{a b}, \quad(a, b=1,2, \ldots, N),
$$

where $\sigma_{A_{a}}$ is the uncertainty of $A_{a}, \delta$ is the Kronecker delta $\left(\delta_{a b}=1\right.$ if $a=b$ and $\delta_{a b}=0$ if $\left.a \neq b\right)$, and $\kappa$ is the GP kernel. Considering the nonparametric nature of our problem, we adopt an exponential squared kernel function defined as

$$
\kappa_{a b}=\sigma_{e}^{2} \delta_{a b}+\sigma_{f}^{2} \exp \left[-\left(\frac{P_{1 a}-P_{1 b}}{\ell_{0}}\right)^{2}-\left(\frac{i_{a}-i_{b}}{\ell_{1}}\right)^{2}\right],
$$

where $P_{1}$ is the main principal component, $i$ is inclination, and $\sigma_{e}, \sigma_{f}, \ell_{0}$, and $\ell_{1}$ are the hyperparameters of the chosen kernel. Training this GP model involves tuning the kernel hyperparameters by maximizing the probability of $\boldsymbol{A}$ to be drawn from the prior distribution, $\mathcal{N}(0, \mathcal{K})$. Similar to Equation (14), the logarithm of likelihood has the following form:

$$
\log \mathcal{L}(\boldsymbol{A})=-\frac{1}{2} \boldsymbol{A}^{T} \mathcal{K}^{-1} \boldsymbol{A}-\frac{1}{2} \log |\mathcal{K}|-\frac{N}{2} \log (2 \pi) .
$$

Adopting the above likelihood and following the same approach explained in Section 4.2, we sample the posterior distribution of the hyperparameters by performing MCMC simulations with 64 chains, each randomly initialized and sampled 10,000 times. We ignore the first 1000 samples of each chain to ensure that they are converged. Table 5 lists the

\footnotetext{
15 http://ned.ipac.caltech.edu/
}

Table 5

Optimized Hyperparameters for the Inverse Squared GP Kernel Defined in Equation (20) to Model the Inclination-dependent Dust Attenuation in Spirals, $A_{\lambda J}^{(i)}$, at Optical Wavelengths

\begin{tabular}{lcccc}
\hline \hline$\lambda$ & $\log \left(\ell_{0}\right)$ & $\log \left(\ell_{1}\right)$ & $\log \left(\sigma_{f}^{2}\right)$ & $\sigma_{e}^{2}$ \\
\hline$u$ & $3.79_{-0.50}^{+0.58}$ & $6.94_{-0.76}^{+1.09}$ & $0.90_{-0.85}^{+1.11}$ & $0.165 \pm 0.005$ \\
$g$ & $3.26_{-0.42}^{+0.49}$ & $5.82_{-0.58}^{+0.65}$ & $-0.31_{-0.77}^{+1.00}$ & $0.110 \pm 0.004$ \\
$r$ & $3.08_{-0.41}^{+0.47}$ & $5.43_{-0.55}^{+0.53}$ & $-0.88_{-0.74}^{+0.96}$ & $0.097 \pm 0.003$ \\
$i$ & $2.91_{-0.41}^{+0.47}$ & $5.17_{-0.57}^{+0.55}$ & $-1.39_{-0.94}^{+0.71}$ & $0.099 \pm 0.003$ \\
$z$ & $2.86_{-0.43}^{+0.48}$ & $4.93_{-0.63}^{+0.76}$ & $-1.90_{-0.68}^{+0.89}$ & $0.099 \pm 0.003$ \\
\hline
\end{tabular}

optimized hyperparameters and their uncertainty bounds after merging all sampled chains.

After finding the functional form of the GP model by optimizing its kernel hyperparameters based on the data of $N$ galaxies with the parameters $\boldsymbol{A}, \boldsymbol{P}_{1}$, and $\boldsymbol{i}$, we need a formalism to predict $\boldsymbol{A}_{*}$ for a set of $M$ galaxies with measured $\boldsymbol{P}_{1 *}$ and $\boldsymbol{i}_{*}$. For simplicity we define $\boldsymbol{X}$ and $\boldsymbol{X}_{*}$ to be the input parameters defined as $\left(\boldsymbol{P}_{1}, \boldsymbol{i}\right)$ and $\left(\boldsymbol{P}_{1 *}, \boldsymbol{i}_{*}\right)$, respectively. Based on the GP assumption, the joint probability of $\boldsymbol{A}$ and $\boldsymbol{A}_{*}$ should follow the same Gaussian distribution; therefore, $\boldsymbol{A}, \boldsymbol{A}_{*} \mid \boldsymbol{X}, \boldsymbol{X}_{*} \sim \mathcal{N}\left(0, \mathcal{K}_{+}\right)$, where $\mathcal{K}_{+}$could be expressed as

$$
\mathcal{K}_{+}=\left(\begin{array}{cc}
\mathcal{K}(\boldsymbol{X}, \boldsymbol{X}) & \mathcal{K}\left(\boldsymbol{X}, \boldsymbol{X}_{*}\right) \\
\mathcal{K}\left(\boldsymbol{X}_{*}, \boldsymbol{X}\right) & \mathcal{K}\left(\boldsymbol{X}_{*}, \boldsymbol{X}_{*}\right)
\end{array}\right)
$$

where $\mathcal{K}(\boldsymbol{X}, \boldsymbol{X})$ is $N \times N, \mathcal{K}\left(\boldsymbol{X}_{*}, \boldsymbol{X}_{*}\right)$ is $M \times M$, and $\mathcal{K}\left(\boldsymbol{X}, \boldsymbol{X}_{*}\right)$ is $N \times M$ and equals $\left(\mathcal{K}\left(\boldsymbol{X}_{*}, \boldsymbol{X}\right)\right)^{T} . \boldsymbol{A}_{*} \mid \boldsymbol{A}, \boldsymbol{X}, \boldsymbol{X}_{*}$ also follows a Gaussian distribution $\mathcal{N}\left(\mu^{*}, \Sigma^{*}\right)$, where its mean and covariance matrix are given by

$$
\begin{aligned}
\mu^{*} & =\mathcal{K}\left(\boldsymbol{X}_{*}, \boldsymbol{X}\right)(\mathcal{K}(\boldsymbol{X}, \boldsymbol{X}))^{-1} \boldsymbol{A}, \\
\Sigma^{*} & =\mathcal{K}\left(\boldsymbol{X}_{*}, \boldsymbol{X}_{*}\right)-\mathcal{K}\left(\boldsymbol{X}_{*}, \boldsymbol{X}\right)(\mathcal{K}(\boldsymbol{X}, \boldsymbol{X}))^{-1} \mathcal{K}\left(\boldsymbol{X}, \boldsymbol{X}_{*}\right) .
\end{aligned}
$$

We use the George Python package developed by Ambikasaran et al. (2015) to facilitate the calculations of the kernel matrix defined by Equation (20) and to make predictions based on Equation (23). The right panel of Figure 8 displays the prediction of our resulting GP model for $A_{r, W_{2}}^{(i)}$.

\section{Appendix B \\ Dust Attenuation as a Function of Infrared Surface Brightness}

In Sections 3.1 and 3.2, we study the correlations between various distance-independent features and optical-infrared color, $\bar{m}_{\lambda}-\bar{W} j$. Throughout the paper, we conducted our analysis of dust attenuation using the main principal component, $P_{1}$, based on its strongest correlation with opticalinfrared colors. We used $P_{1}$ as a proxy that effectively includes the influential physical property of spirals of importance in our study. Calculation of $P_{1}$ requires having knowledge of the H I line profile and its flux. Our sample spirals are chosen to have observations at $21 \mathrm{~cm}$ wavelength for the purpose of distance measurements. In our study, H I information is an essential ingredient in the calculation of dust attenuation. However, the $21 \mathrm{~cm}$ profile/flux might not be available for an arbitrarily chosen spiral. Here, we attempt to present an empirical function for dust attenuation that solely relies on optical and infrared photometric information.

The strong correlation between effective surface brightness, $\left\langle\mu_{2}\right\rangle_{e}^{(i)}, \bar{r}-\bar{W} 2$, and $C_{21 W 2}$ (Figure 3) motivates us to search for 


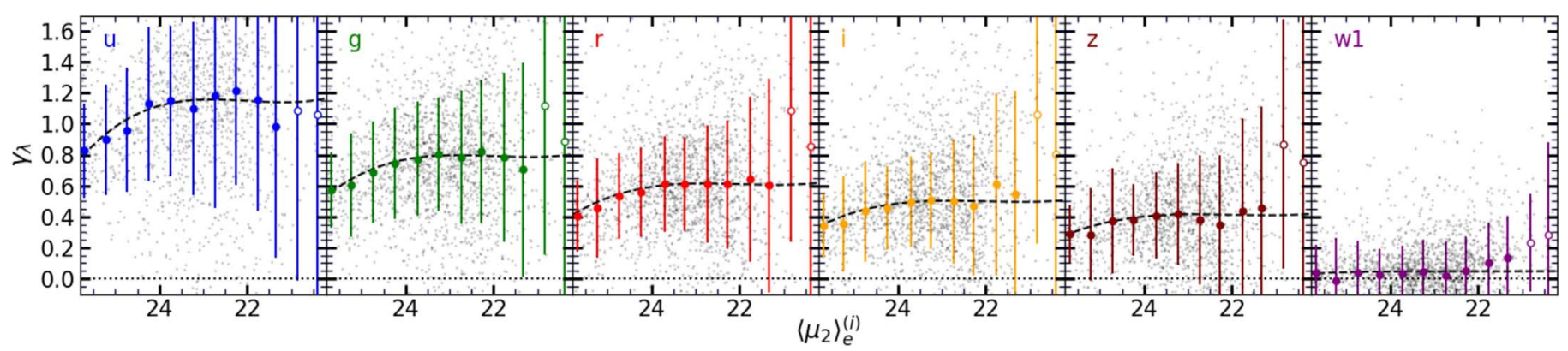

Figure 13. Similar to Figure 10, dust extinction, $\gamma_{\lambda, W 2}$, vs. the $W 2$ effective surface brightness, $\left\langle\mu_{2}\right\rangle_{e}^{(i)}$. Each gray dot represents a spiral whose $\gamma_{\lambda}$ is from Equations (8)-(11). The color points display the average location of galaxies within surface brightness bins with the size of 0.5 mag, and open symbols represent surface brightness values brighter than $21 \mathrm{mag}$. Black curves are the best-fitted third-degree polynomials to gray points that are given by Equation (24).

a relationship between dust attenuation and infrared surface brightness. Figure 13 display $\gamma_{\lambda}$ versus $\left\langle\mu_{2}\right)_{e}^{(i)}$ at different wavebands. Given $P_{1}$ for each spiral, $\gamma_{\lambda}$ is calculated following Equation (12) with parameters taken from Table 4. Figures 10 and 13 motivate us that the average dependency of $\gamma_{\lambda}$ on the physical observables of spirals could possibly be described using similar functions in different wavebands. With that in mind and for the sake of simplicity, we adopt this relation for the dust extinction: $\gamma_{\lambda} \triangleq \rho_{\lambda} G$, where $G$ is a function of infrared surface brightness and has the same form at all bands, and $\rho_{\lambda}$ is the wavelength-dependent dust attenuation factor.

We use a third-degree polynomial function to describe $G$. To obtain more robust results and to improve the statistics, our fitting procedure includes all data points in multiple $u, g, r, i, z$ and $W 1$ bands simultaneously. Our optimized relation between $\gamma_{\lambda}$ and the effective surface brightness of spirals in $W 2$ band is given as

$$
\begin{aligned}
\gamma_{\lambda, W 2}= & \rho_{\lambda}\left(-5.4407 \times 10^{-3}\left\langle\mu_{2}\right\rangle_{e}^{3}\right. \\
& \left.+0.35885\left\langle\mu_{2}\right\rangle_{e}^{2}-7.8782\left\langle\mu_{2}\right\rangle_{e}^{2}+58.363\right),
\end{aligned}
$$

where $\left\langle\mu_{2}\right\rangle_{e}$ is the inclination-corrected effective surface brightness and $\rho_{\lambda} \simeq\left(\gamma_{\lambda} / \gamma_{g}\right)_{a v}$ is the dust extinction factor normalized to $g$ band (see Section 5). The values of $\rho_{\lambda}$ for $u, r$, $i, z$, and $W 1$ bands are $1.45 \pm 0.37,0.77 \pm 0.16,0.63 \pm 0.23$, $0.52 \pm 0.23$, and $0.06 \pm 0.23$, respectively (see Figure 11).

\section{Appendix C \\ $W 1$ versus $W 2$ Principal Components}

One of the most important parameters in our analysis is the main principal component that can be derived based on the photometry of either $W 1$ or $W 2$ bands. Up to this point in this paper, dust obscuration in optical bands and $W 1$ band is derived using the $P_{1, W 2}$ parameter, which is believed to not be significantly under the influence of dust attenuation. Although the effect of dust obscuration is very small on W1-band fluxes, it is not negligible. Despite the small slope of the fiducial relation for the $\bar{W} 1-\bar{W} 2$ color (Figure 5) that suggests the possibility of obtaining the same estimation for the inclinationdependent attenuation following the same analysis using $P_{1, W 1}$ instead of $P_{1, W 2}$, the outcome might be biased. In the top row of Figure 14, we plot the difference between the outputs of our parametric model for dust attenuation that are derived based on the $W 1$ - and $W 2$-band luminosities, i.e., $A_{\lambda, W 1}^{(i)}$ and $A_{\lambda, W 2}^{(i)}$, versus inclination. As expected, this deviation becomes more pronounced as inclination increases because W1-band luminosities are still prone to dust obscuration in more edge-on spirals. Looking at the $W 1$ panels of Figure 6, one might notice this minor inclination dependency of the deviation of $\bar{W} 1-\bar{W} 2$ colors from the corresponding fiducial relation.

On the other hand, the higher quality of photometric data at $W 1$ band motivates us to build a framework based on W1-band photometry that does not suffer such an inclination-dependent bias. Thus, it is more reasonable to use the same formalism we obtained for the $W 2$ band that uses $P_{1, W 2}$.

Figure 15 plots $P_{1, W 1}$ versus $P_{1, W 2}$ for all sample spirals, suggesting a linear relation that allows us to estimate the $W 2$ principal component based on W1-band photometric data. The best-fitted relation is $\left[P_{1, W 2}\right]=1.021 P_{1, W 1}-0.094$, where $\left[P_{1, W 2}\right]$ represents the approximated value of $P_{1, W 2}$. We feed the estimated $\left[P_{1, W 2}\right]$ to the formalism generated based on $W 2$ band to determine the dust attenuation, $\left[A_{W 2}^{(i)}\right]$. In the bottom row of Figure 14, we plot the difference between this new estimation of attenuation, which relies on the $W 1$ band, and $A_{W 2}^{(i)}$ versus inclination. For all wavelengths, the rms scatter of deviations does not show any significant inclination-dependent variations. Over all inclinations the scatter of deviations is less than $\sim 0.03 \mathrm{mag}$, with larger scatter for more edge-on galaxies. 


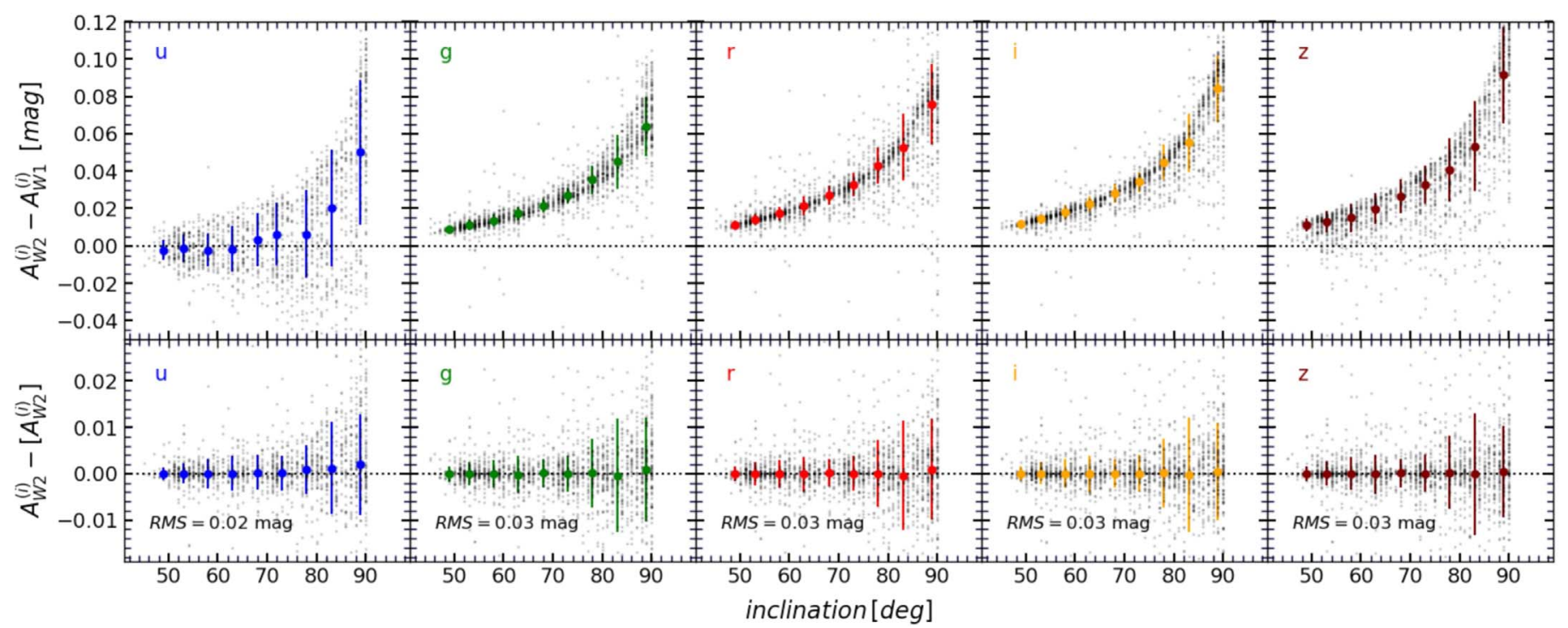

Figure 14. Top row: discrepancy between the inclination-dependent attenuation in spirals calculated based on $W 1$ - and $W 2$-band main principal components, $A_{W 2}^{(i)}-A_{W 1}^{(i)}$, vs. inclination in different optical bands. Bottom row: same as the top row, but for the difference between $A_{W 2}^{(i)}$ and the estimated dust attenuation, $\left[A_{W 2}^{(i)}\right]$, calculated using the linear relation presented in Figure 15 to derive $P_{1, W 2}$ from $P_{1, W 1}$. Each gray dot represents a galaxy, and the points with error bars show the average location of galaxies within inclination bins with the size of $5^{\circ}$, where error bars show $1 \sigma$ scatter of the vertical position of galaxies within each bin.

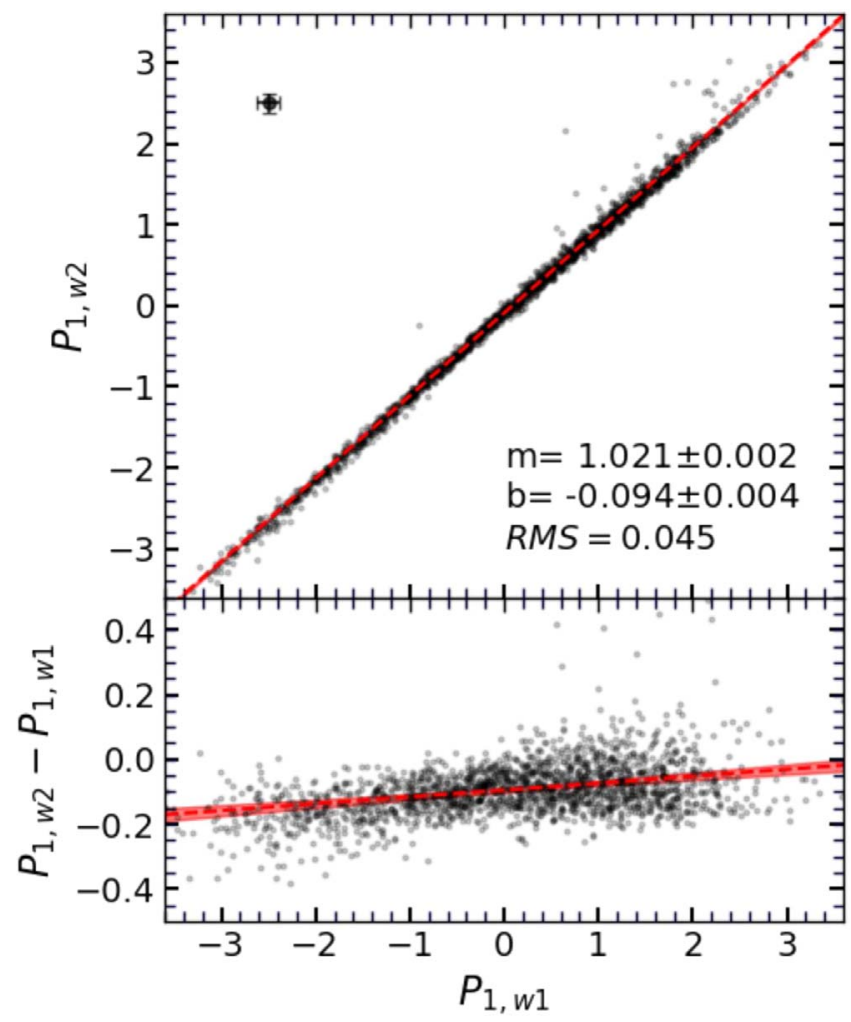

Figure 15. First principal component calculated using $W 2$-band photometric data, $P_{1, W 2}$, vs. that obtained from the W1-band images, $P_{1, W 1}$. Each black dot represents a galaxy with inclination $>45^{\circ}$. The red line shows the best-fitted line with the slope $m$ and intercept $b$. The rms of deviations of black points from the fitted line is calculated along the vertical axis, $P_{1, W 2}$.

\section{ORCID iDs}

Ehsan Kourkchi (1) https://orcid.org/0000-0002-5514-3354

R. Brent Tully (1) https://orcid.org/0000-0002-9291-1981

Mark Seibert (다 https://orcid.org/0000-0002-1143-5515

\section{References}

Ambikasaran, S., Foreman-Mackey, D., Greengard, L., Hogg, D. W., \& O'Neil, M. 2015, ITPAM, 38, 252

Bertin, E., \& Arnouts, S. 1996, A\&AS, 117, 393

Bothwell, M. S., Maiolino, R., Peng, Y., et al. 2016, MNRAS, 455, 1156

Bottinelli, L., Gouguenheim, L., Paturel, G., \& Teerikorpi, P. 1995, A\&A, 296, 64

Cardelli, J. A., Clayton, G. C., \& Mathis, J. S. 1989, ApJ, 345, 245

Chilingarian, I. V., Melchior, A.-L., \& Zolotukhin, I. Y. 2010, MNRAS, 405, 1409

Cho, J., \& Park, C. 2009, ApJ, 693, 1045

Courtois, H. M., \& Tully, R. B. 2012, ApJ, 749, 174

Courtois, H. M., Tully, R. B., Fisher, J. R., et al. 2009, AJ, 138, 1938

Courtois, H. M., Tully, R. B., Makarov, D. I., et al. 2011, MNRAS, 414, 2005

de Vaucouleurs, G., de Vaucouleurs, A., Corwin, H. G., Jr., et al. 1991, Third Reference Catalogue of Bright Galaxies, Vol. I, II, III (New York: Springer)

Draine, B. T. 2011, Physics of the Interstellar and Intergalactic Medium (Princeton, NJ: Princeton Univ. Press)

Feldmann, R. 2015, MNRAS, 449, 3274

Fitzpatrick, E. L. 1999, PASP, 111, 63

Foreman-Mackey, D., Hogg, D. W., Lang, D., \& Goodman, J. 2013, PASP, 125,306

Gibson, N. P., Aigrain, S., Roberts, S., et al. 2012, MNRAS, 419, 2683

Giovanelli, R., Haynes, M. P., Salzer, J. J., et al. 1995, AJ, 110, 1059

Gordon, K. D., Clayton, G. C., Misselt, K. A., Landolt, A. U., \& Wolff, M. J. 2003, ApJ, 594, 279

Han, M. 1992, ApJ, 391, 617

Haynes, M. P., Giovanelli, R., Kent, B. R., et al. 2018, ApJ, 861, 49

Haynes, M. P., Giovanelli, R., Martin, A. M., et al. 2011, AJ, 142, 170

Huang, J.-S., Ashby, M. L. N., Barmby, P., et al. 2007, ApJ, 664, 840

Inoue, A. K. 2011, EP\&S, 63, 1027

Jacob, J. C., Katz, D. S., Berriman, G. B., et al. 2010, Montage: An Astronomical Image Mosaicking Toolkit, Astrophysics Source Code Library, ascl:1010.036

Kent, S. M. 1985, ApJS, 59, 115

Kudritzki, R.-P., Ho, I.-T., Schruba, A., et al. 2015, MNRAS, 450, 342

Law, K.-H., Gordon, K. D., \& Misselt, K. A. 2018, ApJS, 236, 32

Leroy, A. K., Bolatto, A., Gordon, K., et al. 2011, ApJ, 737, 12

Marassi, S., Schneider, R., Limongi, M., et al. 2019, MNRAS, 484, 2587

Masci, F. 2013, ICORE: Image Co-addition with Optional Resolution Enhancement, Astrophysics Source Code Library, ascl:1302.010

Masci, F. J., \& Fowler, J. W. 2009, in ASP Conf. Ser. 411, Astronomical Data Analysis Software and Systems XVIII, ed. D. A. Bohlender, D. Durand, \& P. Dowler (San Francisco, CA: ASP), 67

Masters, K. L., Giovanelli, R., \& Haynes, M. P. 2003, AJ, 126, 158 
Masters, K. L., Nichol, R., Bamford, S., et al. 2010, MNRAS, 404, 792

Neill, J. D., Seibert, M., Tully, R. B., et al. 2014, ApJ, 792, 129

Oke, J. B., \& Sandage, A. 1968, ApJ, 154, 21

Paturel, G., Petit, C., Prugniel, P., et al. 2003, A\&A, 412, 45

Peeples, M. S., \& Shankar, F. 2011, MNRAS, 417, 2962

Rasmussen, C. E., \& Williams, C. K. I. 2006, Gaussian Processes for Machine Learning (Cambridge, MA: MIT Press)

Rezaei, Kh. S., Bailer-Jones, C. A. L., Hanson, R. J., \& Fouesneau, M. 2017, A\&A, 598, A125

Salim, S., Boquien, M., \& Lee, J. C. 2018, ApJ, 859, 11

Salo, H., Laurikainen, E., Laine, J., et al. 2015, ApJS, 219, 4

Schlafly, E. F., \& Finkbeiner, D. P. 2011, ApJ, 737, 103

Schlegel, D. J., Finkbeiner, D. P., \& Davis, M. 1998, ApJ, 500, 525

Springob, C. M., Haynes, M. P., Giovanelli, R., \& Kent, B. R. 2005, ApJS, 160,149
Tremonti, C. A., Heckman, T. M., Kauffmann, G., et al. 2004, ApJ, 613, 898 Tully, R. B., \& Courtois, H. M. 2012, ApJ, 749, 78

Tully, R. B., Courtois, H. M., \& Sorce, J. G. 2016, AJ, 152, 50

Tully, R. B., \& Fisher, J. R. 1977, A\&A, 54, 661

Tully, R. B., \& Pierce, M. J. 2000, ApJ, 533, 744

Tully, R. B., Pierce, M. J., Huang, J.-S., et al. 1998, AJ, 115, 2264

Tully, R. B., Verheijen, M. A. W., Pierce, M. J., Huang, J.-S., \& Wainscoat, R. J. 1996, AJ, 112, 2471

Unterborn, C. T., \& Ryden, B. S. 2008, ApJ, 687, 976

Wright, E. L., Eisenhardt, P. R. M., Mainzer, A. K., et al. 2010, AJ, 140, 1868

Xiao, T., Wang, T., Wang, H., et al. 2012, MNRAS, 421, 486

Yates, R. M., Kauffmann, G., \& Guo, Q. 2012, MNRAS, 422, 215

Yip, C.-W., Szalay, A. S., Wyse, R. F. G., et al. 2010, ApJ, 709, 780

York, D. G., Adelman, J., Anderson, J. E., Jr., et al. 2000, AJ, 120, 1579

Zahid, H. J., Dima, G. I., Kudritzki, R.-P., et al. 2014, ApJ, 791, 130 